\title{
Tough and Osteocompatible Calcium Phosphate Cements Reinforced with Poly(vinyl alcohol) Fibers
}

Nathan W. Kucko, ${ }^{\dagger \neq}, \|$ Sónia de Lacerda Schickert, ${ }^{\dagger, \|}$ Tomás Sobral Marques, $^{\dagger}$ Ralf-Peter Herber, ${ }^{\ddagger}$ Jeroen J. J. P. van den Beuken, ${ }^{\dagger}{ }^{+}$Yi Zuo $^{\S}{ }^{\circledR}$ and Sander C. G. Leeuwenburgh ${ }^{*}{ }^{\dagger}$ (1)

${ }^{\dagger}$ Department of Regenerative Biomaterials, Radboud University Medical Center, Philips van Leydenlaan 25 6525 EX, Nijmegen, The Netherlands

${ }^{\ddagger}$ CAM Bioceramics B.V., Zernikedreef 62333 CL, Leiden, The Netherlands

${ }^{\S}$ Research Center for Nano Biomaterials, Analytical \& Testing Center, Sichuan University 610064 Chengdu, China

\section{Supporting Information}

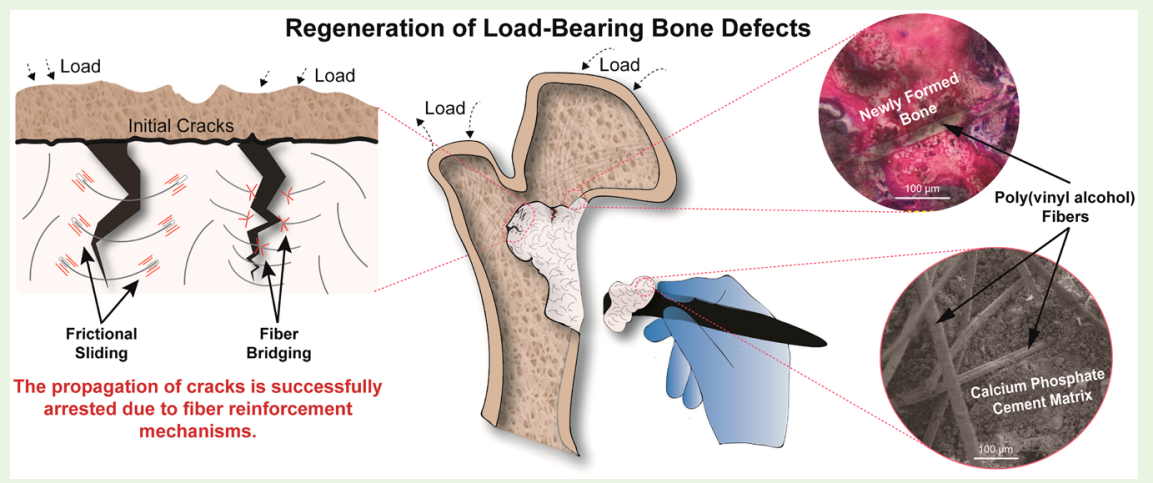

ABSTRACT: Injectable, self-setting calcium phosphate cements (CPCs) are favorable bone substitutes due to their osteocompatibility. However, due to their brittleness and low toughness, their clinical application is limited to non-load-bearing sites. The incorporation of poly(vinyl alcohol) (PVA) fibers into cementitious materials is a successful strategy in civil engineering for improving the mechanical performance of cements. However, PVA fibers in particular have not yet been applied to reinforce CPCs. Therefore, the aim of this study is to investigate the effect of PVA fibers on the mechanical properties of CPCs. Second, the in vitro cytocompatibility of these fibers is studied using cell culture tests. Finally, the in vivo osteocompatibility of PVA fiber-reinforced CPCs is studied after a 6 and 12 week implantation period in the femoral condyle of rabbits. Results reveal that the incorporation of PVA fibers into CPCs is a highly effective strategy to strengthen and toughen CPCs, since the flexural strength and toughness of CPCs increased by more than 3-fold and 435-fold, respectively, upon reinforcement with PVA fibers. In vitro cytocompatibility tests indicate that PVA fibers are cytocompatible, which is further confirmed by the in vivo results that show that PVA fibers do not compromise the excellent osteocompatibility of CPCs.

KEYWORDS: calcium phosphate cements, fiber-reinforcement, biomedical applications, poly(vinyl alcohol) fibers,

mechanical properties

\section{INTRODUCTION}

Calcium phosphate cements (CPCs) are a class of bioceramics characterized as injectable, self-setting bone substitute materials. Apatite-forming CPCs share a chemical composition similar to that of the inorganic component (i.e., hydroxyapatite) found in native bone, providing them with favorable osteocompatible properties. $^{1-5}$ These characteristics, coupled with the fact that CPCs are injectable, set under physiological conditions, and can be utilized in minimally invasive surgical procedures, ${ }^{6,7}$ has allowed CPCs to stand out as an attractive alternative to other routine bone healing treatments (e.g., autografts) and other conventional bone substitute materials (e.g., bioceramic granules)., 8
Since their discovery in the early 1980s by Brown and Chow, ${ }^{10}$ CPCs have undergone significant research in an effort to improve their clinical handling, biodegradation, and mechanical properties. Nevertheless, CPCs still lack the mechanical properties needed to be utilized in load-bearing anatomical sites due to their inherent brittleness and poor strength and toughness when compared to those of native bone. ${ }^{5,11-13}$ This, in turn, has severely restricted their clinical applicability to surgeries such as tibial plateau augmentation and the treatment of certain maxillo-craniofacial defects, all of

Received: February 13, 2019

Accepted: April 16, 2019

Published: April 16, 2019 
which are mainly loaded in compression where CPCs can withstand greater loads as compared to tension where they are more prone to failure. ${ }^{3,11,13}$

Successful improvement of the mechanical strength and especially toughness of CPCs would significantly expand their clinical applicability ${ }^{5,12}$ to more load-bearing defect sites such as vertebroplasty, kyphoplasty, ${ }^{8,9,14}$ long bone replacement, ${ }^{15}$ and fixation of articulating prostheses. ${ }^{16}$ Therefore, several strategies have been used in an attempt to reduce the brittleness and increase the toughness of CPCs, including both intrinsic and extrinsic approaches. ${ }^{2,5,17}$ Some of the most common approaches include (1) reducing the porosity of the CPC matrix, ${ }^{17,18}$ (2) the use of a dual setting cement system involving the cross-linking of polymeric monomers, ${ }^{19,20}$ and (3) the reinforcement with fibers, ${ }^{2-5,12,16}$ with the latter being the most popular and successful approach to date. This strategy has been adopted from traditional fields of engineering such as civil engineering, where fiber-reinforcement of concretes and hydraulic cements to improve their mechanical integrity has been extensively used for building purposes. ${ }^{21}$ Various types of fibers have been incorporated into CPCs in the form of inorganic whiskers ${ }^{22,23}$ as well as degradable and nondegradable polymeric fibers ${ }^{2,3,12}$ in either short ${ }^{16,24}$ or long, discontinuous lengths. ${ }^{17,25}$

The mechanical performance of fiber-reinforced CPCs is dictated by a complex interaction between the various constituents of the composite material which involves (i) fiber bridging, (ii) crack deflection, and (iii) frictional sliding. Fiber bridging becomes operational when the matrix begins to crack under an applied load and the fibers bridge the crack together, effectively arresting further crack propagation. Crack deflection occurs when fibers act as a barrier for cracks to grow and in doing so prolongs the distance at which the crack needs to travel through the matrix, resulting in more energy dissipation. $^{21,26,29}$ Frictional sliding transpires when fibers are pulled out of the matrix against frictional stress at the fibermatrix interface. This phenomenon allows for a stress transfer whereby the load applied on the matrix is dissipated to the fibers, effectively absorbing more energy. ${ }^{28}$

Several key parameters determine the effectiveness of the above-mentioned fiber-reinforcing mechanisms and mechanical performance of the resulting CPCs including fiber aspect ratio, concentration, orientation and dispersion, tensile strength and elastic modulus, biocompatibility, and affinity toward the matrix. $^{2,12}$ Within CPC research, the importance of the fiber-matrix affinity has been poorly addressed. ${ }^{2,17}$ Biodegradable polyester-based fibers have been used most extensively to reinforce CPCs. Nevertheless, these fibers are mechanically weak and hydrophobic, which compromises their affinity for hydrophilic cement matrices. Consequently, typical fiber-reinforcement efficacies for CPCs are poor as compared to reinforcement of cementitious matrices in civil engineering. ${ }^{2,17}$ In civil engineering, fibers made of poly(vinyl alcohol) (PVA) are frequently used to improve the strength and toughness of cementitious matrices in view of their favorable mechanical properties, hydrophilicity, and strong affinity for the inorganic matrix. ${ }^{28-30}$ However, these fibers have not yet been used for reinforcement of bioceramic cements. Therefore, we hypothesize that reinforcement of CPCs with PVA fibers allows for substantial improvement of the reinforcing efficacy of CPCs without harming the favorable biological performance of these bioceramic materials.
Specifically, the hydrophilic nature of PVA fibers should allow for a strong affinity with hydrophilic CPCs. ${ }^{28-30}$ In addition, PVA fibers are stronger and stiffer when compared to other nondegradable fibers used for reinforcement of CPCs like nylon, polypropylene, and polyethylene. ${ }^{30}$ Finally, PVA is regarded as a biocompatible polymer in the biomedical field, as evidenced by its use in biomedical applications that range from hydrogels used in ophthalmic applications to meniscus and cartilage replacements in orthopedics. ${ }^{31,32}$ Previously, PVA has been added as a soluble nonfibrous additive to CPCs, but PVA fibers have never been incorporated into CPCs. ${ }^{33}$

In the present study, we investigate the effect of PVA fibers on the mechanical and biological properties of CPCs by varying the fiber concentration (2.5-5 wt \%) and length (3-6 $\mathrm{mm}$ ). Two types of commercially available PVA fibers with or without a hydrophobic surface coating were selected to decipher how the fiber-matrix affinity influences these mechanical and biological properties. As CPCs are inherently brittle, they are weaker under tensile and shear forces which occur at load-bearing sites within the body. Generally, compression testing is not recommended for mechanical testing of the performance of fiber-reinforced CPCs. ${ }^{2}$ Therefore, CPCs were subjected to a standard three-point flexural test to measure their flexural strength, flexural modulus, and work-of-fracture (as a measure for toughness) since these properties are key factors in predicting the load-bearing capacity of CPCs. ${ }^{3}$ Further, an in vitro cytocompatibility test was performed on the PVA fibers to determine their cytocompatibility. Finally, the osteocompatibility of both resorbable and nonresorbable PVA fiber-reinforced CPCs was assessed upon implantation in the femoral condyle of rabbits.

\section{MATERIALS AND METHODS}

2.1. Preparation of Fiber-Reinforced Calcium Phosphate Cements. The powder phase of the calcium phosphate cement (CPC) consisted of $100 \% \alpha$-tricalcium phosphate kindly provided by CAM Bioceramics B.V. (Leiden, The Netherlands), while the liquid phase consisted of a 4 wt $\% \mathrm{NaH}_{2} \mathrm{PO}_{4} \cdot 2 \mathrm{H}_{2} \mathrm{O}$ (Merck, Darmstadt, Germany) aqueous solution. Two commercially available poly(vinyl alcohol) (PVA) fibers were selected for this study: (i) PVA Kura $_{\text {fibers }}$ kindly provided by Kuraray Europe $\mathrm{GmbH}$ (Kuralon, item \#RMS702/6 mm, Hattersheim am Main, Germany), and (ii) PVA $_{\text {Mini }}$ fibers kindly provided by MiniFIBERS Inc. (PVOH, Johnson City, TN, USA). PVA ${ }_{\text {Mini }}$ fibers were hydrophilic in nature due to the absence of any surface modification, whereas PVA $_{\text {Kura }}$ fibers were modified with a proprietary hydrophobic coating.

Fabrication of fiber-reinforced CPCs was initiated by adding PVA fibers to the powder phase of the cement in amounts specified in Table 1. Next, the liquid phase was added to the powder phase at a

Table 1. PVA Fiber Specifications

\begin{tabular}{ccccc} 
fiber type & $\begin{array}{c}\text { fiber amount } \\
{[\text { wt \% }]}\end{array}$ & $\begin{array}{c}\text { length } \\
{[\mathrm{mm}]}\end{array}$ & $\begin{array}{c}\text { fiber } \\
\text { diameter } \\
{[\mu \mathrm{m}]}\end{array}$ & $\begin{array}{c}\text { aspect ratio } \\
{[\text { length/diameter] }}\end{array}$ \\
PVA $_{\text {Kura }}$ & 2.5 and 5.0 & 3 and 6 & 26 & 115 and 231 \\
PVA $_{\text {Mini }}$ & 2.5 and 5.0 & 3 and 6 & 14 & 214 and 429 \\
\hline
\end{tabular}

liquid-to-powder ratio of 1:2. The phases were then thoroughly mixed together for $1 \mathrm{~min}$ until a cementitious paste was formed. Subsequently, the paste was placed in polydimethylsiloxane (PDMS) molds $(25 \times 4 \times 4 \mathrm{~mm})$, clamped between two glass slides and allowed to set for $24 \mathrm{~h}$ at room temperature. After setting, the specimens were immersed in phosphate-buffered saline solution (PBS) and placed on a shaker table set to $120 \mathrm{rpm}$ in an incubator at 
$37^{\circ} \mathrm{C}$ for $72 \mathrm{~h}$ to allow the $\mathrm{CPC}$ to fully cure. Mechanical testing was performed immediately after removing the cement specimens from the PBS solution in order to ensure testing was performed under wet conditions.

2.2. Characterization of CPC and PVA Fibers. 2.2.1. Powder $X$-ray Diffraction. A PANalytical X'Pert ${ }^{3}$ powder X-ray diffractometer (Malvern Panalytical B.V., Eindhoven, The Netherlands) with a $\mathrm{Cu}$ $\mathrm{K} \alpha$ radiation source was employed to perform powder $\mathrm{X}$-ray diffraction (XRD) on CPC before and after the setting reaction as specified in section 2.1 in order to characterize the phase transformation. Further, XRD was also performed on $\mathrm{PVA}_{\text {Kura }}$ and $\mathrm{PVA}_{\text {Mini }}$ fibers to compare their crystallinity. The measurements were performed at a voltage and current of $45 \mathrm{kV}$ and $40 \mathrm{~mA}$, respectively, with a step size of $0.05^{\circ}$ and a count time of $3.0 \mathrm{~s}$ per step. For the fiber-free CPC specimens, the scan was from $20^{\circ}$ to $60^{\circ} 2 \theta$, and for the PVA fibers, the scan was from $10^{\circ}$ to $40^{\circ} 2 \theta$.

2.2.2. Fourier Transform Infrared Spectroscopy. Fourier transform infrared spectroscopy (FTIR, Spectrum One, PerkinElmer Inc., Waltham, Massachusetts, USA) was performed on $\mathrm{PVA}_{\mathrm{Kura}}$ and $\mathrm{PVA}_{\text {Mini }}$ fibers, and their representative spectra were compared with one another to characterize any chemical differences. Spectra were measured under attenuated total reflectance (ATR) with a spectral resolution of $4 \mathrm{~cm}^{-1}$ at 4 scans per measurement. Prior to each measurement, a background scan was performed in open air at room temperature. Finally, each spectrum was normalized by performing a ATR and baseline correction followed by smoothing using Spectrum 10 software (PerkinElmer Inc.).

2.3. Mechanical Testing. A universal testing machine (Lloyd Instruments Ltd., Bognor Regis, UK) was used to perform a standard three-point flexural test on the fiber-reinforced CPC specimens using a support span of $20 \mathrm{~mm}$. A $100 \mathrm{~N}$ loading cell was used with a crosshead speed of $1 \mathrm{~mm} / \mathrm{min}$ for fiber-reinforced cements and 0.2 $\mathrm{mm} / \mathrm{min}$ for fiber-free cements. The crosshead speed was increased for fiber-reinforced CPCs because the crack growth for these specimens was slower which can interfere with the measurement. Therefore, it is recommended in ASTM C1161-02C-Standard Test Method for Flexural Strength of Advanced Ceramics at Ambient Temperature that a crosshead speed of $1.0 \mathrm{~mm} / \mathrm{min}$ should be used when this is the case. The flexural strength $(S)$, flexural modulus $(E)$, and work-of-fracture (WOF) were then measured from the loaddisplacement curves as described in eqs 1,2 , and 3, respectively. There were 10 specimens measured for each CPC composition.

$$
\text { flexural strength: } S=\frac{3 P_{\max } L}{2 b h^{2}}
$$

In this equation, $P_{\max }$ represents the maximum load on the loaddisplacement curve, $L$ is the support span, and $b$ and $h$ indicate the specimen's width and height, respectively. ${ }^{4,34}$

$$
\text { flexural modulus: } E=\frac{m L^{3}}{4 b h^{3}}
$$

In this equation, $m$ indicates the slope of the line tangent to the linearelastic portion of the load-displacement curve. ${ }^{4,34}$

$$
\text { work-of-fracture: } \mathrm{WOF}=\frac{A}{b h}
$$

In this equation, $A$ indicates the total area under the loaddisplacement curve. In the literature, work-of-fracture is commonly referred to as the toughness of the material and is typically measured in kilojoules per meter squared $\left(\mathrm{kJ} \mathrm{m}^{-2}\right)$. In order to allow for a consistent calculation of the WOF, all tests were stopped at a displacement of $3 \mathrm{~mm}$.

2.4. In Vitro Cytocompatibility Assay. The cytocompatibility of both $\mathrm{PVA}_{\mathrm{Mini}}$ and $\mathrm{PVA}_{\text {Kura }}$ fibers was assessed by measuring the proliferation of osteoblastic cells (MC3T3-E1; ATCC, USA) cultured in the presence or absence of different concentrations of PVA fibers. A fiber-free group consisting of osteoblastic cells cultured in the absence of PVA fibers was also included as a positive control. The selected PVA fiber concentrations ranged between 0.1 and $3 \mathrm{mg} \mathrm{mL}^{-1}$. Fibers were weighed in Transwell permeable supports $(4 \mu \mathrm{m}$ pore size, Merck KGaA, Darmstadt, Germany), after which each support was immersed in $70 \%$ ethanol $(\mathrm{v} / \mathrm{v})$ for $15 \mathrm{~min}$, followed by two washing cycles using sterilized PBS. MC3T3-E1 cells were cultured in $\alpha$ minimum essential medium ( $\alpha$-MEM, supplemented with $10 \%$ fetal bovine serum and $1 \%$ Gentamicin, Gibco, Life Technologies Grand Island, USA). Cells were seeded at a density of 10000 cells $\mathrm{cm}^{-2}$ in polystyrene 12 well-plates and cultured for $24,48,72$, and $168 \mathrm{~h}$ (medium was replaced after $72 \mathrm{~h}$ ). After each time point, the medium and transwell supports were discarded and cells were washed twice using PBS, followed by the addition of Milli-Q water $(2 \mathrm{~mL}$ per well $)$ and 2 freeze cycles. Cellular DNA content was measured using a Quant-IT PicoGreen ds-DNA assay kit (Invitrogen; Breda, The Netherlands) according to the manufacturer's instructions. Briefly, samples or standard solution $(100 \mu \mathrm{L})$ was added to a working solution in a 96-well plate. The plates were then incubated for $5 \mathrm{~min}$ in the dark at room temperature, and the fluorescence emission was read (excitation, $485 / 20 \mathrm{~nm}$; emission, $530 / 25 \mathrm{~nm}$ ).

2.5. In Vivo Biocompatibility Assay. All animal experiments were conducted in the Central Animal Laboratory at Sichuan University, China. The protocols were approved by the Animal Ethical Committee of Sichuan University and were performed according to the Chinese national guidelines for the care and use of laboratory animals. Healthy, skeletally mature, male New Zealand white rabbits, weighing $3 \pm 0.5 \mathrm{~kg}$, were used for this study. The rabbits were housed separately and given 1 week to acclimate to the animal laboratory, after which the surgeries were performed. The animals were fed with rabbit chow and water ad libitum throughout the study period along with close monitoring for any clinical signs of discomfort (e.g., weight loss, distress, or behavioral alterations).

2.5.1. Preparation of Materials for Implantation. Four experimental groups were selected as described in Table 2. Poly(D,L-lactic-

Table 2. Compositions of the Solid Phase of Implanted CPCs

\begin{tabular}{lccc}
\multicolumn{1}{c}{ implant type } & $\begin{array}{c}\text { CPC } \\
\text { [wt \%] }\end{array}$ & $\begin{array}{c}\text { PLGA } \\
\text { [wt \%] }\end{array}$ & $\begin{array}{c}\text { PVA fibers [wt \%] } \\
\text { (6 mm in length) }\end{array}$ \\
fiber-free CPC & 100.0 & & \\
CPC/PLGA & 60.0 & 40.0 & \\
CPC/PLGA/PVA $_{\text {Mini }}$ & 58.5 & 39.0 & 2.5 \\
CPC/PVA $_{\text {Mini }}$ & 97.5 & 0.0 & 2.5 \\
\hline
\end{tabular}

co-glycolic acid) particles (PLGA, lactic/glycolic acid ratio of 50/50; molecular weight of $17 \mathrm{kDa}$; acid-terminated, Corbion Purac B.V., Gorinchem, The Netherlands) were included in two experimental groups as porogens to accelerate the degradation of the CPC matrix, as demonstrated previously. ${ }^{35-37}$ Preset cylindrical specimens $(5 \times 5$ $\mathrm{mm}^{2}$ ) were prepared by using the same preparation method used for the mechanical tests. After preparation, the specimens were immersed in PBS and sterilized with ethylene oxide (Synergy Health, Venlo, The Netherlands).

2.5.2. Surgical Implantation Procedure. Each rabbit was subjected to a one-session surgical procedure, in which two formulations were randomly implanted bilaterally (i.e., one in each femoral condyle). Randomization was ensured by distributing the experimental groups over the recipient sites per implantation period according to a randomization scheme. All surgical procedures were performed with the animals under general anesthesia, induced by ear vein injection of 3\% sodium pentobarbital (Sinopharm Chemical Reagent Co., Ltd., Shanghai, China), at a dose of $45 \mathrm{mg} \mathrm{kg}^{-1}$. To reduce the risk of perioperative infection, the rabbits received antibiotic prophylaxis 30 min prior to surgery and for 3 days postsurgery, by an intramuscular injection of benzylpenicilin sodium $0.48 \mathrm{~g}$. After anesthesia, rabbits were immobilized on their backs, and the hind limbs were shaved, washed, and disinfected with povidone-iodine. A longitudinal skin incision was performed over the medial femoral condyle, followed by blunt dissection and removal of the periosteum. Once the medial condyle was fully exposed, a trephine drill was used to create a $5 \mathrm{~mm}$ 

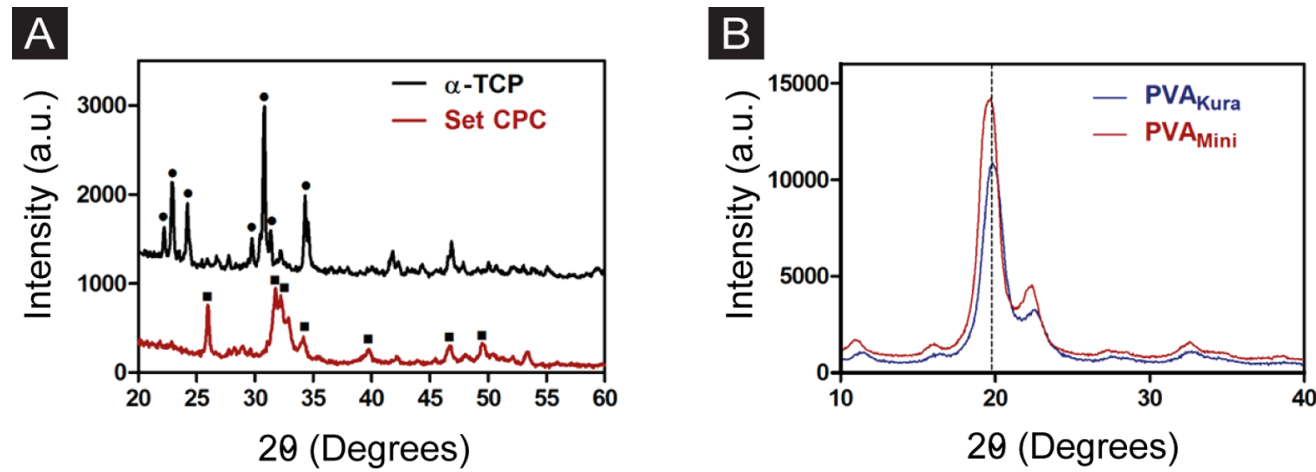

Figure 1. Powder X-ray diffractograms of (A) the CPC powder precursor phase before and after fully setting and (B) PVA-only fibers. The circles in Figure 1A represent diffraction peaks characteristic of $\alpha$-TCP according to Powder Diffraction File ICDD 9-348, and squares represent diffraction peaks characteristic to hydroxyapatite according to Powder Diffraction File ICDD 9-432. The dotted line in Figure $1 \mathrm{~B}$ represents the (101) reflection at $19.4^{\circ} 2 \theta$, indicative of PVA in both $\mathrm{PVA}_{\mathrm{Kura}}$ and $\mathrm{PVA}_{\mathrm{Mini}}$ fibers.

round bone defect to a depth of $5 \mathrm{~mm}$. The preset cylindrical CPC composites from either of the four experimental groups prepared previously were press-fitted into the bone defect. Soft tissues were then closed in separate layers using resorbable single sutures (Vicryl 3-0, Ethicon, Inc., Chaoyang District, Beijing, China). Postoperative analgesia was administered for 2 days by an intramuscular injection of xylazin hydrocholoride (Biochemistry Co. Ltd., Shanghai) at a dose of $0.1 \mathrm{mg} \mathrm{kg}^{-1}$. Finally, after the selected implantation periods of 6 and 12 weeks, animals were sacrificed with an overdose of sodium pentobarbital.

2.5.3. Specimen Retrieval and Histological Processing. After euthanasia, both femora were retrieved and closely analyzed for any adverse reactions in the tissue and surrounding structures. After inspection, the femoral condyle was excised and adhering soft tissues were removed using a diamond saw and a scalpel, respectively. The isolated specimens were then fixated in a $4 \%$ phosphate-buffered formalin solution $(\mathrm{pH} 7.2)$, dehydrated in a graded series of ethanol concentrations $(70-100 \%)$, and finally embedded in poly(methyl methacrylate) (PMMA). After polymerization, three histological sections of $\sim 10 \mu \mathrm{m}$ in thickness were prepared in a cross-sectional direction (i.e., perpendicular to the longitudinal direction of the bone defect) using a saw microtome with a diamond blade (SP 1600, Leica Microsystems AG, Wetzlar, Germany). The sections were stained with methylene blue and basic fuchsine and imaged using a digital slide scanner (VS120-S5; Olympus, Tokyo, Japan) for further analysis.

2.5.4. Histological and Histomorphometrical Analysis. Digital histology images were analyzed according to a standard developed by the International Organization for Standardization (Biological evaluation of medical devices, Part 6-Tests for local effects after implantation; ISO 10993-6). Briefly, this method relies on comparison of the biological response of experimental test specimens to the biological response of control specimens. Control specimens are defined as materials of which clinical acceptability and/or biocompatibility characteristics have been previously established. For this study, fiber-free CPC and CPC/PLGA were considered as control groups for our PVA-reinforced test groups $\mathrm{CPC} / \mathrm{PVA}_{\mathrm{Mini}}$ and $\mathrm{CPC} / \mathrm{PLGA} / \mathrm{PVA}_{\mathrm{Mini}}$, respectively. In more detail, this standard categorizes the biological response in terms of four main biological processes, i.e., inflammation, neovascularization, fibrosis, and/or fatty infiltrate. Four specimens were tested for each experimental or control group $(n=4)$. Scores ranged from 0 to 4 depending on the frequency and/or severity of the observed processes. Subsequently, scores of the four main biological processes were added together. Finally, the average score for the control groups was subtracted from the average score obtained for the experimental test groups. This final total score categorized the experimental test materials as nonirritant (score between 0 and 2.9), slightly irritant (score between 3 and 8.9), moderately irritant (score between 9 and 15), or severely irritant (score exceeding 15). More details on this scoring method derived from ISO 10993-6 can be found in the Supporting Information (Table S1).

Histomorphometrical parameters such as the presence of newly formed bone and material remnants inside the region-of-interest (ROI) were determined quantitatively. To this end, the ROI was defined as the bone defect area that was filled with the preset cylindrical cement ( $5 \mathrm{~mm}$ in diameter), and images were processed using image processing software (ImageJ, National Institutes of Health, Bethesda, Maryland, USA). To define the ROI graphically, a 5 $\mathrm{mm}$ circle was superimposed on each image and centered around the bone defect. The outside of the circle was removed, and the area of newly formed bone, as well as the remaining material, was determined on the basis of color recognition with manual correction and expressed as a percentage of bone or material within the total area of the ROI, respectively.

2.5.5. Crack Length Determination. The total length of cracks detectable inside the ROI of fiber-free CPC and CPC/PVA specimens was measured using ImageJ. Samples containing PLGA were excluded from this analysis since their degradable nature did not allow for a clear detection of cracks. Additionally, preset cylindrical fiber-free CPC and CPC/PVA ${ }_{\text {Mini }}$ control specimens were created and processed in the same way as the implanted specimens. Sections of these nonimplanted specimens were processed and imaged as described above to measure the total crack length per sample prior to implantation.

2.6. Examination of Morphology of Fiber-Reinforced CPCs. 2.6.1. Scanning Electron Microscopy. To evaluate the CPC surface morphology and fiber-matrix interface, fractured specimens were sputter-coated with a $20 \mathrm{~nm}$ thick chromium layer and imaged using a scanning electron microscope (SEM; Zeiss Sigma 300, Zeiss AG, Oberkochen, Germany). PVA fibers were also evaluated using SEM before and after cell culture for $168 \mathrm{~h}$ to decipher any changes in the surface morphology.

2.6.2. Nanocomputed Tomography Analysis. Nanocomputed tomography (nanoCT) was performed on all CPCs selected for in vivo testing to visualize the dispersion of the fibers within the cement matrix. Specimens were scanned using a GE Phoenix Nanotom M nanoCT system, research edition (General Electric, Brussels, Belgium), with the X-ray source parameters having a voltage of 90 $\mathrm{kV}$, a current of $110 \mu \mathrm{m}$, and a focal spot size of $2.34 \mu \mathrm{m}$. Acquisition parameters included an exposure time of $500 \mathrm{~ms}$ that produced an image with a pixel size of $3072 \times 2400$.

2.7. Statistical Analysis. All values from this study were presented as a mean \pm standard deviation. All statistical analysis was conducted using GraphPad Prism software (version 7.0d, GraphPad Software Inc., San Diego, USA) with a level of significance of $p<0.05$.

2.7.1. Analysis of Mechanical Properties. A Grubbs' outlier test (significance level $=0.01$ ) was performed on the WOF values for all specimens, and any outliers were subsequently removed from the data 
A

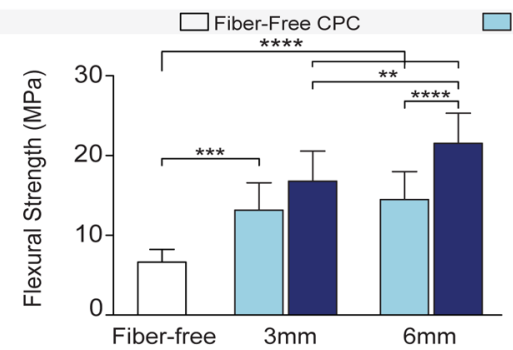

C

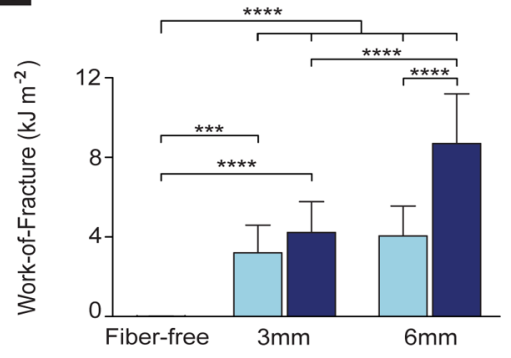

E

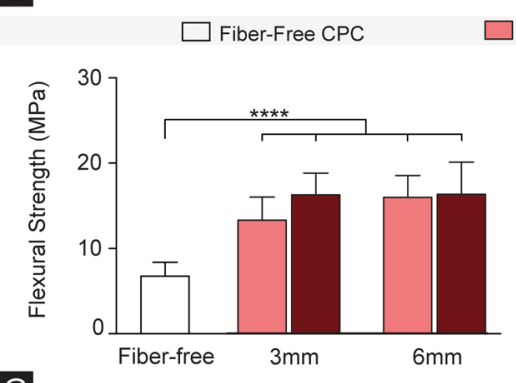

G

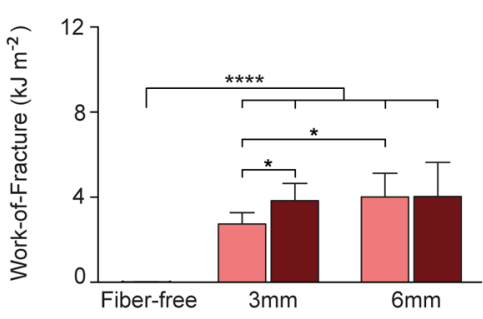

B

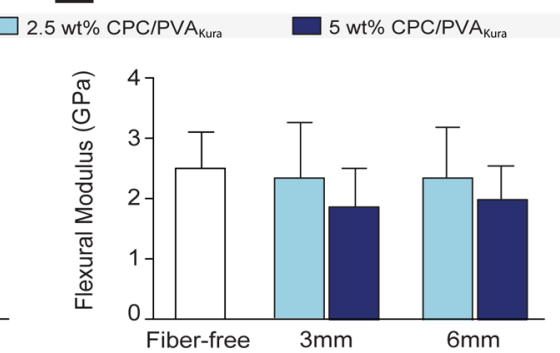

D

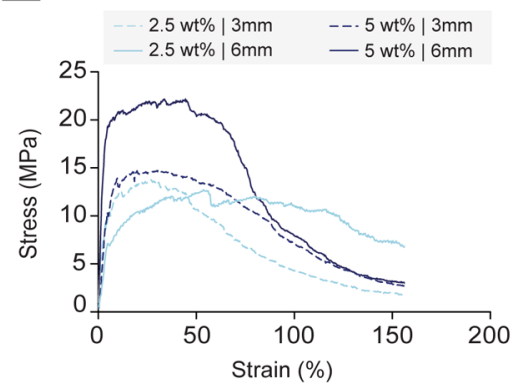

$\mathrm{F}$

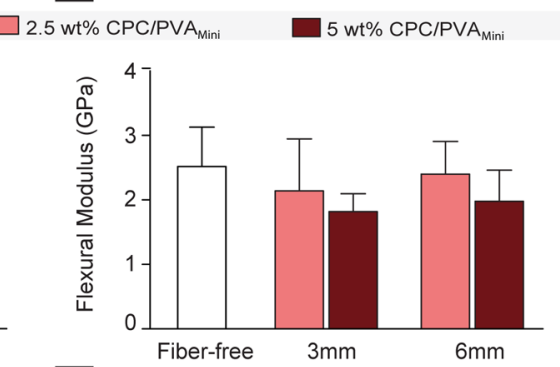

$\mathrm{H}$

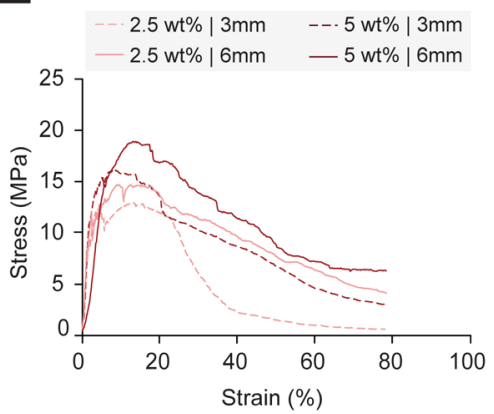

Figure 2. Effect of fiber length and weight concentration on the flexural strength (A, E), flexural modulus (B, F), work-of-fracture (C, G), and representative stress vs strain curves $(D, H)$ of CPCs reinforced with $P_{V A} A_{\text {Kura }}$ fibers $(A-D)$ or $P A_{M i n i}$ fibers $(E-H)$. Significant differences are indicated by $* * p<0.01, * * * p<0.001$, and $* * * * p<0.0001$. For comparative purposes, the work-of-fracture for the fiber-free control specimen was measured as $0.020 \pm 0.008 \mathrm{~kJ} \mathrm{~m}^{-2}$.

set. A two-way analysis of variance (ANOVA) with a posthoc Bonferroni test was used to statistically analyze the mechanical properties of PVA fiber-reinforced CPC groups, whereas a one-way ANOVA with a posthoc Tukey-Kramer multiple comparisons test was used to compare the mechanical properties of the four experimental groups selected for in vivo testing.

2.7.2. Analysis of Cyto- and Osteocompatibility. Statistical analysis of the DNA quantification $(n=6)$ was performed using two-way ANOVA with a posthoc Tukey-Kramer multiple comparisons test, while the histomorphometrical data $(n=3)$ were analyzed using a one-way ANOVA with a Tukey-Kramer multiple comparisons posthoc test. Statistical analysis of the crack length ( $n$ = 3) was performed using a one-way ANOVA with a posthoc Dunnett's multiple comparison test. In addition, the comparison of crack length between nonimplanted $\mathrm{CPC}$ and $\mathrm{CPC} / \mathrm{PVA}_{\mathrm{Mini}}$ specimens was performed using an unpaired $t$ test.

\section{RESULTS}

3.1. Characterization of CPC and PVA Fibers. X-ray diffractograms of the hardened CPC reveal diffraction peaks characteristic of hydroxyapatite (Powder Diffraction File ICDD $9-432)^{38}$ for the fully set CPC, which confirms that the phase transformation from $\alpha$-TCP to hydroxyapatite was complete upon full setting (Figure 1A). Diffractograms of $\mathrm{PVA}_{\text {Kura }}$ and $\mathrm{PVA}_{\mathrm{Mini}}$ fibers are similar in crystallinity, with both showing a pronounced peak at $19.4^{\circ} 2 \theta$ which is indicative of the (101) reflection of PVA (Figure 1B). ${ }^{39}$ 


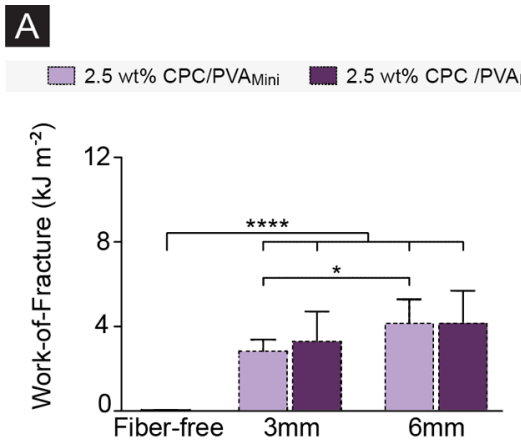

B

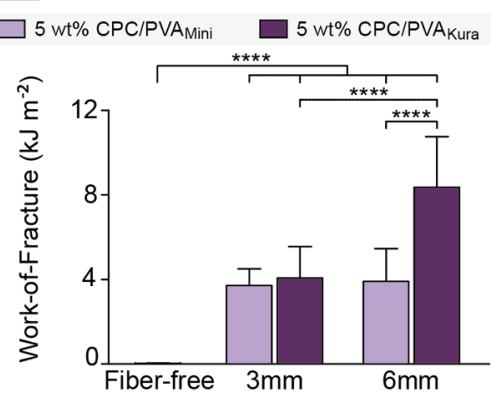

Figure 3. Comparison of work-of-fracture values for $\mathrm{CPC} / \mathrm{PVA}_{\mathrm{Kura}}$ and $\mathrm{CPC} / \mathrm{PVA}_{\mathrm{Mini}}$ groups at 2.5 wt \% (A) and 5 wt \% (B). Significant differences between experimental groups are indicated by $* p<0.05$ and $* * * * p<0.0001$. For comparative purposes, the work-of-fracture for the fiber-free control specimen was measured as $0.020 \pm 0.008 \mathrm{~kJ} \mathrm{~m}^{-2}$.

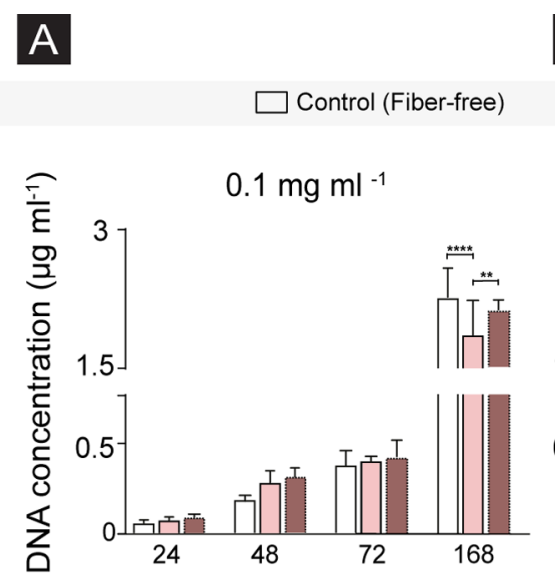

\section{B}

C

$\square$ PVA Fiberkura

$1 \mathrm{mg} \mathrm{ml}^{-1}$

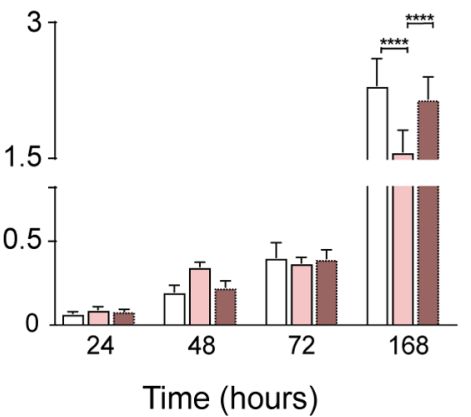

PVA Fibermini

$3 \mathrm{mg} \mathrm{ml}^{-1}$

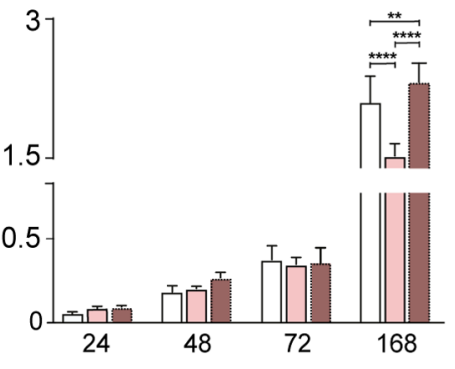

Figure 4. DNA quantification of MC3T3-E1 cells exposed to different concentrations of both PVA $_{\text {Kura }}$ and PVA $A_{\text {Mini }}$ fibers at 0.1 (A), 1 (B), and 3 (C) $\mathrm{mg} \mathrm{mL}^{-1}$ of fibers. The fiber-free group corresponds to a positive control. Significant differences are indicated by $* * p<0.01$ and $* * * * p<$ 0.0001 .

\subsection{Mechanical Properties of PVA Fiber-Reinforced}

CPCs. Figure 2A-D illustrates the effect of fiber length and concentration on the mechanical properties of CPCs reinforced with PVA fibers modified with a hydrophobic surface coating $\left(\mathrm{PVA}_{\text {Kura }}\right.$ Kuraray). Both the fiber length and concentration improved the flexural strength more than 2-fold (Figure 2A), with specimens containing $5 \mathrm{wt} \%$ fibers of $6 \mathrm{~mm}$ in length exhibiting the greatest improvement of $21.5 \pm 3.8$ $\mathrm{MPa}$ compared to $6.6 \pm 1.6 \mathrm{MPa}$ for the fiber-free CPC (Table S2). Further, doubling the fiber concentration from 2.5 to $5 \mathrm{wt}$ $\%$ improved the flexural strength of CPCs for both fiber lengths $(3$ and $6 \mathrm{~mm}$ ), whereas an increase in fiber length from 3 to $6 \mathrm{~mm}$ did not improve the strength of cements containing $2.5 \mathrm{wt} \%$ fibers. This observation suggests that fiber concentration has a more pronounced effect on the flexural strength than fiber length. No statistical difference in the flexural modulus (Figure 2B) was observed for either of the tested groups.

Cement toughness, on the other hand, nearly tripled from $3.2 \pm 1.4$ to $8.7 \pm 2.5 \mathrm{~kJ} \mathrm{~m}^{-2}$ when both the fiber concentration and length increased from $2.5 \mathrm{wt} \% 3 \mathrm{~mm}$ to $5 \mathrm{wt} \% 6 \mathrm{~mm}$ (Figure 2C). Moreover, the toughness for fiberfree CPC specimens measured only $0.020 \pm 0.008 \mathrm{~kJ} \mathrm{~m}^{-2}$, meaning that the addition of $5 \mathrm{wt} \% 6 \mathrm{~mm} \mathrm{PVA}_{\mathrm{Kura}}$ fibers substantially improved the toughness of CPCs by 435 -fold. Interestingly, the absence of a statistical difference among the 5 wt $\% 3 \mathrm{~mm}, 2.5 \mathrm{wt} \% 3 \mathrm{~mm}$, and $2.5 \mathrm{wt} \% 6 \mathrm{~mm}$ groups suggests that a threshold was reached for these fiber conditions. The corresponding stress-strain curves shown in Figure 2D confirm the effective toughening of CPCs using $\mathrm{PVA}_{\mathrm{Kura}}$ fibers, where the area calculated underneath these curves corresponds to the toughness of the CPCs. We observed that the $5 \mathrm{wt} \% 6 \mathrm{~mm}$ group reached a yield stress of nearly $20 \mathrm{MPa}$ before the cement began to deform plastically followed by a strain-hardening phase. Contrary to this behavior, all other groups only reached a stress of about 7-9 MPa before revealing a similar strain-hardening behavior. For ceramics, this strain-hardening behavior is indicative of ductile materials where multiple microcracks form in the cement matrix without inducing complete failure, indicating that the composite cement is able to withstand loads due to several fiber-reinforcement mechanisms. ${ }^{34}$

Figure $2 \mathrm{E}-\mathrm{H}$ shows the mechanical properties of $\mathrm{CPCs}$ reinforced with hydrophilic, unmodified PVA fibers $\left(\mathrm{PVA}_{\mathrm{Mini}}\right.$ MiniFIBERS) fibers. Similar to CPCs reinforced with hydrophobic PVA $_{\text {Kura }}$ fibers, both an increase in the length and concentration of $\mathrm{PVA}_{\mathrm{Mini}}$ fibers contributed to an improvement in the flexural strength and toughness, where the $5 \mathrm{wt} \% 6 \mathrm{~mm}$ group represented the highest values of $16.1 \pm 3.7 \mathrm{MPa}$ and $4.1 \pm 1.6 \mathrm{~kJ} \mathrm{~m}^{-2}$ for flexural strength and toughness (Figure $2 \mathrm{E}, \mathrm{G})$, respectively. Interestingly, the flexural strength values for both the $\mathrm{PVA}_{\mathrm{Kura}}$ and $\mathrm{PVA}_{\text {Mini }}$ fiber-containing CPCs fall 
A

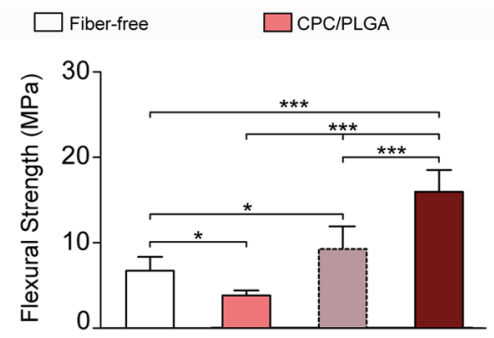

C

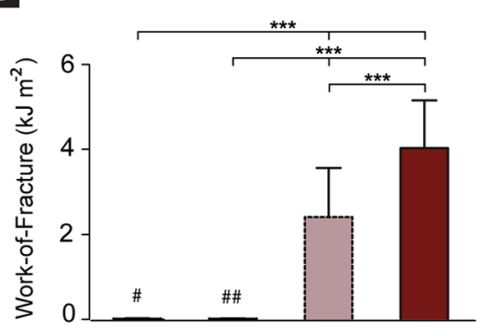

B

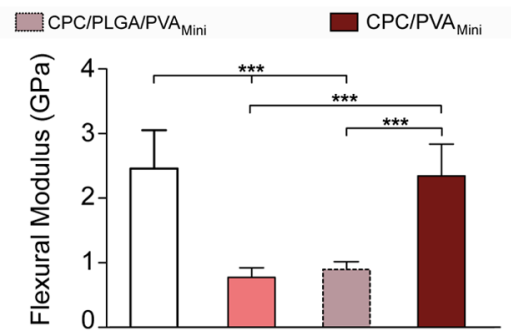

D

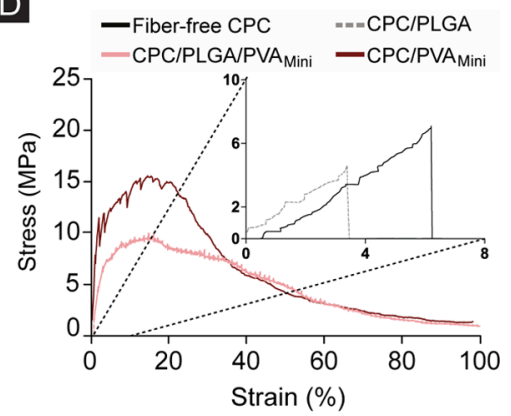

Figure 5. Comparison of the flexural strength (A), flexural modulus (B), work-of-fracture (C), and representative stress vs strain curves (D) of the four experimental groups selected for osseous implantation. Significant differences are indicated by $* p<0.05$ and $* * * p<0.001$. For comparative purposes, the work-of-fracture values for the fiber-free control $\left(^{\#}\right)$ and fiber-free CPC/PLGA $\left({ }^{\# \#}\right)$ specimens were measured as $0.020 \pm 0.008$ and $0.020 \pm 0.004 \mathrm{~kJ} \mathrm{~m}^{-2}$, respectively.

within the range reported for trabecular bone (10-20 MPa), irrespective of fiber length or concentration. ${ }^{40}$ Again, the flexural modulus of CPCs was hardly affected by the incorporation of $\mathrm{PVA}_{\mathrm{Mini}}$ fibers (Figure $2 \mathrm{~F}$ ).

The stress-strain curves illustrated in Figure $2 \mathrm{H}$ shows a similar pattern as observed for CPCs reinforced with PVA $_{\text {Kura }}$ fibers, where a maximum stress was reached at relatively low strains followed by plastic deformation. However, after this initial yield (i.e., stress at which plastic deformation occurs and the matrix starts developing cracks), the stress drops at a faster rate under lower strains when compared to CPCs reinforced with PVA $_{\text {Kura }}$ fibers. This behavior is indicative of tensionsoftening and, consequently, results in lower toughness values. These differences become more evident in Figure 3, where the toughness of CPCs reinforced with either $\mathrm{PVA}_{\text {Kura }}$ or $\mathrm{PVA}_{\mathrm{Mini}}$ fibers is compared. The toughness of CPCs reinforced with 5 wt $\%$ of $6 \mathrm{~mm}$ long $\mathrm{PVA}_{\mathrm{Kura}}$ fibers $\left(8.7 \pm 2.5 \mathrm{~kJ} \mathrm{~m}^{-2}\right)$ was more than 2-fold higher than that of CPCs reinforced with the same concentration of $6 \mathrm{~mm}$ long $\mathrm{PVA}_{\mathrm{Mini}}$ fibers $\left(4.1 \pm 1.6 \mathrm{~kJ} \mathrm{~m}^{-2}\right)$. CPCs reinforced with hydrophobically modified PVA $_{\text {Kura }}$ fibers displayed a strain-hardening behavior where the maximum flexural strength was observed at $\sim 50 \%$ strain (Figure 2D), whereas CPCs reinforced with hydrophilic PVA $_{\text {Mini }}$ fibers revealed a tension-softening behavior with maximum strength values observed at a strain of only $\sim 15 \%$ (Figure $2 \mathrm{H}$ ). A summary of the flexural strength, flexural modulus, and toughness for all CPC groups can be found in Table S2.

3.3. Cytocompatibility of PVA Fibers. To assess the cytocompatibility of the selected PVA fibers, a DNA quantification assay was performed for MC3T3-E1 cells cultured in the presence of $\mathrm{PVA}_{\mathrm{Mini}}$ or $\mathrm{PVA}_{\mathrm{Kura}}$ fibers at concentrations of $0.1,1$, and $3 \mathrm{mg} \mathrm{mL}^{-1}$. MC3T3-E1 cells were also cultured in the absence of fibers (fiber-free) as a positive control. Figure 4 shows that the DNA content increased with time for both types of fibers. Up to $72 \mathrm{~h}$ of culture, cell proliferation was equal for all experimental groups, while the proliferation of cells cultured for $168 \mathrm{~h}$ in the presence of PVA $_{\text {Kura }}$ fibers was lower than fiber-free controls. PVA ${ }_{\text {Mini }}$ fibers did not reduce the proliferation of the cultured cells at any of the time points or fiber concentrations. The morphology of both $\mathrm{PVA}_{\mathrm{Kura}}$ and $\mathrm{PVA}_{\mathrm{Mini}}$ fibers was not affected by cell culture, as shown by SEM images in Figure S2 of the Supporting Information.

3.4. Osseous Implantation of PVA Fiber-Reinforced CPCs. Due to their superior cytocompatibility (Figure 4), $\mathrm{PVA}_{\text {Mini }}$ fibers were selected for further testing of the osteocompatibility of PVA fiber-reinforced CPCs using an established femoral condyle implantation model in rabbits. A $\mathrm{PVA}_{\text {Mini }}$ fiber concentration of $2.5 \mathrm{wt} \%$ was selected since a higher fiber concentration did not result in higher toughness values for CPCs reinforced with PVA $_{\text {Mini }}$ fibers. Further, a fiber length of $6 \mathrm{~mm}$ was selected since the toughness was significantly higher $(* p<0.05)$ when compared to a CPC reinforced with the same fiber concentration but with $\mathrm{PVA}_{\mathrm{Mini}}$ fibers $3 \mathrm{~mm}$ in length (Figure 3 ). The effect of PVA $_{\text {Mini }}$ fibers on the osteocompatibility of CPCs was studied for apatitic CPCs which were either resorbable due to the incorporation of porogens made of poly(D,L-lactic-co-glycolic acid) (PLGA) porogens, or left nonresorbable (PLGA-free CPC). The abbreviations and compositions of the four selected experimental groups, i.e., (i) fiber- and PLGA-free CPC, (ii) fiber-

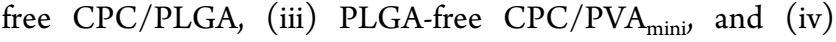
$\mathrm{CPC} / \mathrm{PLGA} / \mathrm{PVA}_{\mathrm{Mini}}$ are provided in Table 2. Prior to implantation, the mechanical properties (Figure 5) and morphology of the fractured specimens (Figure S1) were characterized in more detail. Movies produced from 3D reconstructed nanoCT images were also employed to evaluate the dispersion of fibers and PLGA microparticles (Movies S1S4). SEM micrographs along with nanoCT videos indicated that PLGA microparticles were dispersed homogeneously 
6 WEEKS
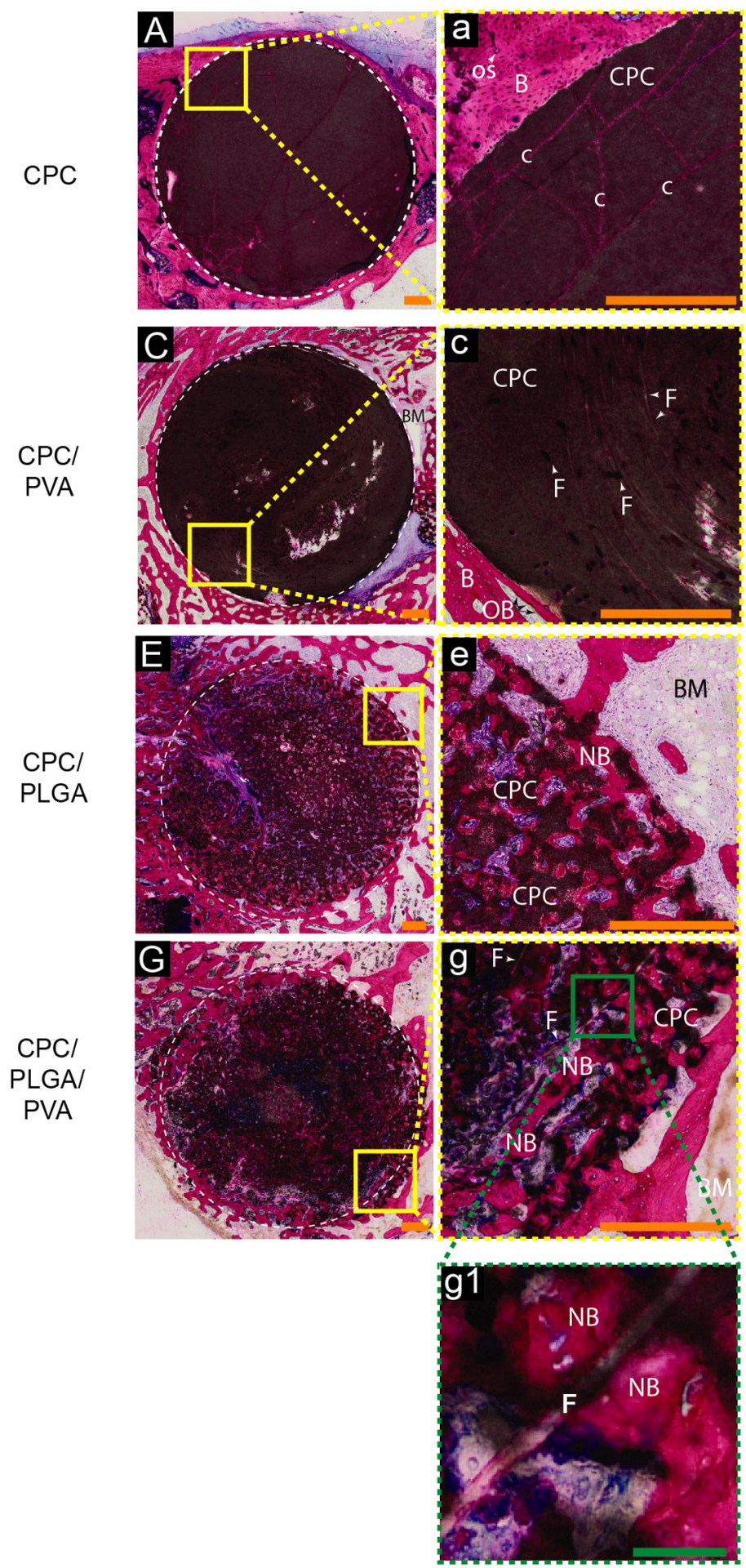

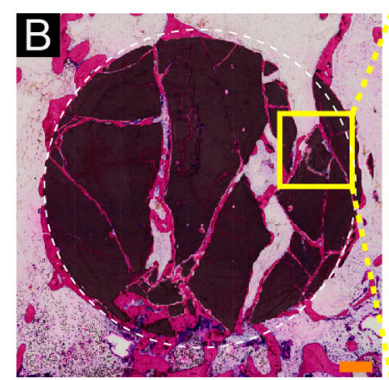

12 WEEKS
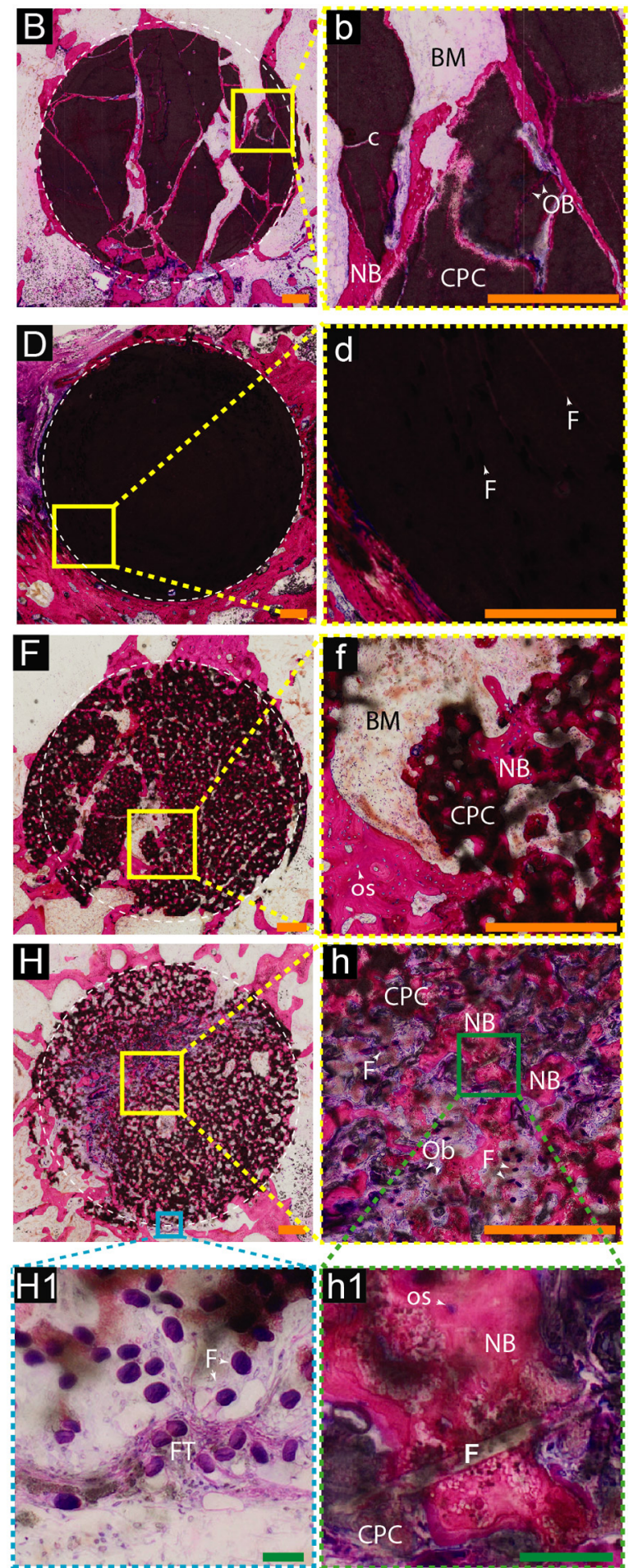

Figure 6. Representative histological images of fiber-free CPC, CPC/PVA $\mathrm{Mini}_{\text {, }} \mathrm{CPC} / \mathrm{PLGA} / \mathrm{PVA} \mathrm{M}_{\mathrm{Mini}}$, and CPC/PLGA after 6 and 12 weeks of osseous implantation in the femoral condyle of rabbits. Lowercase letters are magnifications of the corresponding uppercase letters, while $\mathrm{H} 1$, h1, and $\mathrm{g} 1$ are magnifications of $\mathrm{H}, \mathrm{h}$, and g, respectively. The ROI is indicated by a white dashed circle. Orange scale bars correspond to $1000 \mu \mathrm{m}$, and green scale bars correspond to $100 \mu \mathrm{m}$. Legend: OS, osteocytes; OB, osteoblasts; CPC, remaining cement; c, cracks in the cement matrix; BM, bone marrow; NB, newly formed bone; F, $\mathrm{PVA}_{\mathrm{Mini}}$ fibers; FT, fibrous tissue.

throughout the matrix (Figure S1A-B, Movies S1 and S2) whereas fibers tended to aggregate, particularly at higher concentrations of fibers (Movie S4). SEM micrographs also revealed substantial differences in the fracture plane and fibermatrix interface between $\mathrm{CPC} / \mathrm{PVA}_{\mathrm{Mini}}$ and $\mathrm{CPC} / \mathrm{PVA}_{\mathrm{Kura}}$ specimens. Unlike $\mathrm{CPC} / \mathrm{PVA}_{\mathrm{Mini}}$ specimens, the fracture plane of $\mathrm{CPC} / \mathrm{PVA}_{\mathrm{Kura}}$ specimens show fibers protruding from the matrix along with the presence of fiber-shaped empty tunnels (Figure S1D1,D2). Further, PVA $_{\text {Kura }}$ fibers exhibited surface abrasion whereas $\mathrm{PVA}_{\mathrm{Mini}}$ fibers were abundantly 
coated with CPC particles, which suggests that unmodified PVA $_{\text {Mini }}$ fibers had a stronger affinity to the matrix than PVA $_{\text {Kura }}$ fibers which were pulled out from the matrix (Figure S1C3,D3). These observations suggest that frictional resistance against fiber pullout was lower for CPCs reinforced with PVA $_{\text {Kura }}$ fibers.

Generally, the addition of PLGA porogens reduced the flexural strength and modulus of CPCs considerably (Figure $5 \mathrm{~A}, \mathrm{~B})$. Conversely, the addition of $\mathrm{PVA}_{\mathrm{Mini}}$ fibers improved the flexural strength to $9.1 \pm 2.6$ and $15.8 \pm 2.5 \mathrm{MPa}$ for CPC/ PLGA/PVA ${ }_{\mathrm{Mini}}$ and $\mathrm{CPC} / \mathrm{PVA}_{\mathrm{Mini}}$ groups, respectively. The toughness of CPCs increases substantially upon the incorporation of $\mathrm{PVA}_{\mathrm{Mini}}$ fibers up to $2.4 \pm 1.2$ and $4.0 \pm 1.1 \mathrm{~kJ} \mathrm{~m}^{-2}$ for $\mathrm{CPC} / \mathrm{PLGA} / \mathrm{PVA}_{\mathrm{Mini}}$ and $\mathrm{CPC} / \mathrm{PVA}_{\mathrm{Mini}}$ cements, respectively (Figure $5 \mathrm{C}$ ). Figure $5 \mathrm{D}$ revealed that fiber-free cements (i.e., CPC and CPC/PLGA) experienced a brittle fracture mode characterized by very low strain-to-failure $(\sim 3-6 \%)$ and toughness values $\left(\sim 0.020 \mathrm{~kJ} \mathrm{~m}^{-2}\right)$. In contrast, fiber-reinforced cements (i.e., CPC/PLGA/PVA ${ }_{M i n i}$ and $\mathrm{CPC} / \mathrm{PVA}_{\mathrm{Mini}}$ ) exhibited stress-strain curves indicative of quasibrittle materials revealing tension-softening behavior.

Both the surgery and the postoperative periods were uneventful for all animals. During the retrieval of the specimens, after 6 and 12 weeks, no macroscopically adverse tissue reactions were observed. An overview of the histological images of all groups at both implantation periods is provided in Figure 6. PLGA-free CPCs did not degrade, which limited the ingrowth of new bone as observed in Figure 6A-D. In contrast, PLGA-containing CPCs resorbed with time, resulting in replacement of these CPCs with new bone (Figure 6E-H). All groups showed intimate contact between the implanted cement and the surrounding bone. Necrosis or alterations in tissue morphology provoked by an adverse inflammatory reaction were not observed. However, an inflammatory response was detected around large agglomerates of $\mathrm{PVA}_{\mathrm{Mini}}$ fibers for the CPC/PLGA/PVA ${ }_{\text {Mini }}$ group (Figure $6 \mathrm{H} 1$ ), characterized by narrow bands of fibrosis and a minimal amount of fatty infiltrate. Polymorphonuclear inflammatory cells were, however, hardly observed. In order to quantify the biological response of fiber-containing CPCs relative to fiberfree CPCs as controls, a semiquantitative scoring system (Table S1) was used. The scoring results of this semiquantitative evaluation can be found in Table 3, which indicated that both experimental groups can be classified as nonirritant materials.

The amounts of CPC retention and newly formed bone in the region-of-interest (ROI) were quantified histomorphometrically as shown in Figure 7A,B. PLGA-free CPCs did not degrade, resulting in almost $100 \%$ retention of $\mathrm{CPC}$, while

Table 3. Semiquantitative Evaluation of the Biological Response of PVA Fiber-Reinforced CPCs after Osseous Implantation $^{a}$

\begin{tabular}{llcc} 
& & \multicolumn{2}{c}{ scoring results } \\
\cline { 3 - 4 } experimental group & control group & 6 weeks & 12 weeks \\
CPC/PVA $_{\mathrm{Mini}}$ & fiber-free CPC & $1.3 \pm 2.6$ & $0.0 \pm 2.3$ \\
$\mathrm{CPC} / \mathrm{PLGA} / \mathrm{PVA}_{\mathrm{Mini}}$ & $\mathrm{CPC} / \mathrm{PLGA}$ & $1.8 \pm 1.9$ & $0.5 \pm 1.3$
\end{tabular}

${ }^{a}$ Definitions: nonirritant (0.0-2.9), slight irritant (3.0-8.9), moderate irritant $(9.0-15.0)$, severe irritant $(>15.0)$. More details on this scoring method derived from ISO 10993-6 can be found in the Supporting Information (Table S1).
PLGA-containing CPCs showed retention values of $\sim 50 \%$ after 6 weeks and $\sim 25 \%$ after 12 weeks (Figure 7A). Accordingly, hardly any bone was formed in these PLGAfree CPCs (Figure 7B). In contrast, CPC/PLGA and CPC/

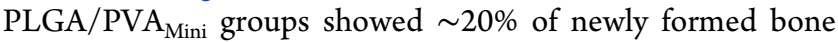
after 6 weeks and $\sim 30 \%$ after 12 weeks (Figure $7 \mathrm{~B}$ ).

Occasionally, cracks were observed in the histological sections of PLGA-free CPCs. To delineate whether these cracks were present before implantation (due to processing of the cement specimens) or if they were formed after implantation, additional studies were performed. The length of these cracks in sections of explanted specimens was quantified in direct comparison to sections of nonimplanted PLGA-free CPCs that were subjected to similar histological processing steps as the implanted specimens (Figure 7C,D). Evidently, cracks were also present in the sections of these nonimplanted specimens (Figure $7 \mathrm{C}$ ), and the length of these cracks was similar to the cracks detected in the implanted specimens (Figure 7D). Generally, crack length was lower for CPCs reinforced with $\mathrm{PVA}_{\mathrm{Mini}}$ fibers for nonimplanted specimens.

\section{DISCUSSION}

PVA fibers were introduced into CPCs to achieve substantial enhancement of their strength and toughness by enabling transfer of loads from the matrix to the fibers. To this end, PVA fibers were selected in view of the biocompatibility and hydrophilicity of PVA as well as the high strength and stiffness of commercially available PVA fibers. To vary the surface chemistry of these PVA fibers, two types of commercially available PVA fibers were tested, i.e., unmodified hydrophilic

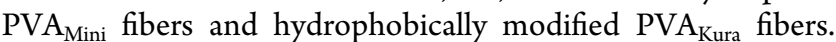
These fibers were added to CPCs at different lengths and weight percentages, and their mechanical properties, morphology, and molecular structure were thoroughly characterized. Further, a cytocompatibility assay was performed to study the in vitro biological response of the selected PVA fibers. Finally, CPCs containing $\mathrm{PVA}_{\mathrm{Mini}}$ fibers and/or PLGA porogens were implanted into the femoral condyle of rabbits in order to evaluate their osteocompatibility.

Typically, CPCs fail catastrophically in a brittle manner without any preceding deformation which is unfavorable when applied to load-bearing anatomical sites. Generally, the degree of ductility and toughness of a material can be measured and categorized into three different modes of failure: (i) brittle, (ii) tension-softening for quasibrittle materials, and (iii) strainhardening for highly ductile materials. As failure modes shift from brittle to tension-softening to strain-hardening, as dictated by the behavior of their stress-strain curves, a significant improvement in the strain-to-failure and toughness can be observed. ${ }^{2}$

Unlike PVA-reinforced CPCs, the stress-strain curves of unreinforced CPCs exhibited brittle failure at low strains (3$6 \%$ ) (Figure 5D) which corresponds to low toughness. ${ }^{12}$ With the addition of PVA $_{\text {Mini }}$ fibers, the stress-strain curves show tension-softening behavior and the strain increases to $15-20 \%$ resulting in improved toughness (Figure $2 \mathrm{G}, \mathrm{H}$ ). The ability of fibers to improve the toughness of CPCs is caused by several factors. The fibers both deflect cracks and bridge cracks together, thereby hindering the propagation of the cracks and dissipating energy throughout the matrix due to the nucleation of new cracks. The amount and density of these microcracks grow with increasing stress until a saturation level is reached 
A

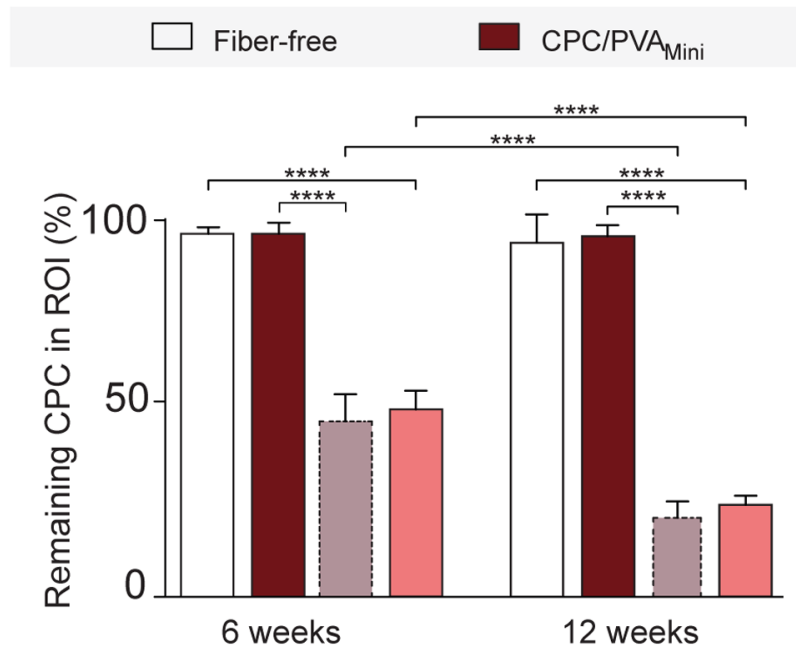

C

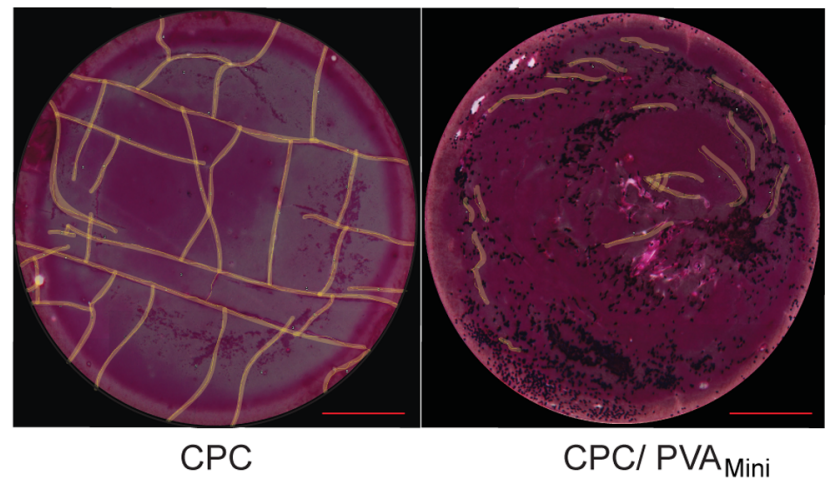

B
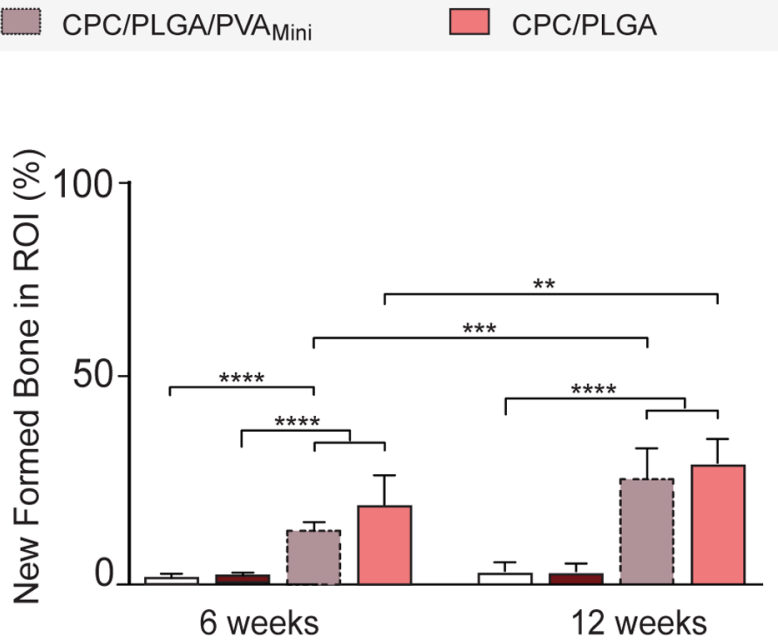

$\mathrm{D}$

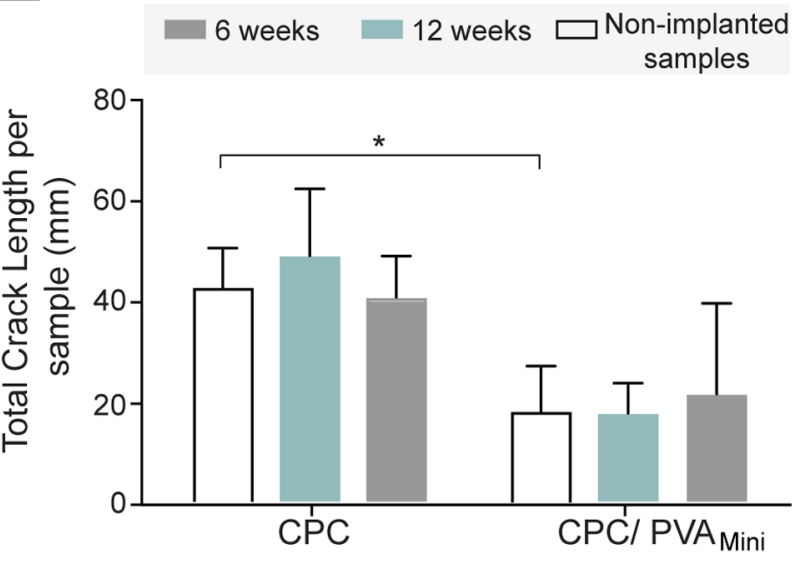

Figure 7. Histomorphometric analysis of the percentage of remaining CPC (A) and percentage of newly formed bone (B) after 6 and 12 weeks of osseous implantation, and representative histological images of cements containing cracks (C) and quantification of the total crack length per specimen (D) for both nonimplanted and implanted specimens. Significant differences are indicated by $* * p<0.01, * * * p<0.001$, and $* * * * p<$ 0.0001 . The red scale bars correspond to $1000 \mu \mathrm{m}$.

after which previously arrested cracks propagate further. ${ }^{41}$ Energy is also absorbed and transferred from the matrix to the fibers through frictional sliding at the fiber-matrix interface. ${ }^{21,27,42}$ Frictional sliding is evidenced by fibers pulling out from the matrix, which was more evident for $\mathrm{CPC} / \mathrm{PVA}_{\text {Kura }}$ cements (Figure S1D1,D2). Moreover, PVA $_{\text {Kura }}$ fibers also displayed surface abrasion which is indicative of the occurrence of the interfacial slip (Figure S1D3) that allowed for efficient dissipation of energy and higher toughness values. ${ }^{2,34}$

The ability of PVA $_{\text {Kura }}$ fibers to prevent crack propagation is greater when compared to PVA $_{\text {Mini }}$ fibers, as evidenced by the shift in the stress-strain curve behavior from tension-softening for $\mathrm{CPC} / \mathrm{PVA}_{\mathrm{Mini}}$ specimens (Figure $2 \mathrm{H}$ ) to strain-hardening for $\mathrm{CPC} / \mathrm{PVA}_{\mathrm{Kura}}$ specimens (Figure $2 \mathrm{D}$ ), resulting in greater CPC toughness (Figure 3). Here, debonding at the fibermatrix interface during frictional sliding is improved due to the hydrophobic coating which allows for more efficient transfer of loads to the fibers. ${ }^{12,28}$ The result is a highly toughened and bendable CPC that revealed extremely high toughness values $\left(8.7 \pm 2.5 \mathrm{~kJ} \mathrm{~m}^{-2}\right)$. These values exceed the highest values reported so far for fiber-reinforced CPCs by Xu et al., where carbon fibers were incorporated into CPCs to obtain toughness values up to $6.5 \mathrm{~kJ} \mathrm{~m}^{-2,2,25}$ which stresses the efficacy of PVA fibers for reinforcement of CPCs. Further, this substantial improvement in the toughness and ductility of CPCs reinforced with PVA fibers can expand their clinical applicability to more load-bearing defect sites where conventional unreinforced CPCs are unable to provide the appropriate mechanical stability that is needed. More specifically, certain procedures such as vertebroplasty, kyphoplasty, ${ }^{8,9,14}$ long bone replacement, ${ }^{15}$ and fixation of articulating prostheses ${ }^{16}$ or dental implants would become areas of interest for PVA fiber-reinforced CPCs.

When comparing the toughness between the two PVAreinforced CPCs, CPCs reinforced with 5 wt \% of hydrophobically modified $\mathrm{PVA}_{\mathrm{Kura}}$ fibers were about 2 times tougher than CPCs reinforced with unmodified hydrophilic $\mathrm{PVA}_{\text {Mini }}$ fibers (Figure 3). We speculate that this observation can be attributed to differences in the fiber hydrophobicity and, consequently, the fiber-matrix affinity of the two fiber types. 
Since stress is transferred from the cement to the fiber during frictional sliding at the fiber-matrix interphase, understanding and tailoring this interphase interaction is of utmost importance in order to engineer highly toughened CPCs. ${ }^{2,12,28,43}$ If the interphase bond is too weak, the fiber will pull out, whereas an excessively strong bond will result in fiber rupture before energy dissipation can occur. ${ }^{26,44} \mathrm{PVA}_{\mathrm{Mini}}$ fibers are inherently hydrophilic due to the presence of surface hydroxyl groups, rendering them more susceptible to fiber rupture rather than frictional sliding as the hydroxyl groups bond strongly to the surrounding matrix. ${ }^{28,30}$ This is evidenced by SEM micrographs of $\mathrm{CPC} / \mathrm{PVA}_{\mathrm{Mini}}$ cements after fracturing (Figure S1C), where fibers do not protrude out of the matrix fracture plane and fiber-shaped tunnels caused by fiber pullout were not observed. This difference suggests that PVA $_{\text {Mini }}$ fibers were primarily ruptured rather than being pulled out from the matrix. Further, the abundant presence of CPC particles on the surface of $\mathrm{PVA}_{\mathrm{Mini}}$ fibers (Figures S1C3) supports the fact that PVA $_{\text {Mini }}$ fibers have a strong affinity to the CPC matrix, thereby compromising the toughening mechanism caused by frictional sliding. Conversely, $\mathrm{PVA}_{\mathrm{Kura}}$ fibers contain a hydrophobic coating designed to reduce their hydrophilicity and, therefore, their affinity with hydraulic cements, allowing for a more efficient stress transfer to transpire. ${ }^{28}$ We speculate that this reduced fiber-matrix affinity caused the improvement in toughness and ductility for CPCs reinforced with hydrophobic PVA $_{\text {Kura }}$ fibers.

Scheffler and co-workers ${ }^{28}$ characterized the chemical composition of this hydrophobic coating using FTIR and reported the presence of ester and amide groups that are attributed to cationic surface-active agents, while the presence of acidic polyethylene was associated with long chain polyethylene waxes. FTIR spectra of PVA $_{\text {Kura }}$ and PVA $_{\text {Mini }}$ fibers (Figure S3) were similar except for the presence of a peak around $2850 \mathrm{~cm}^{-1}$ which was only present in the $\mathrm{PVA}_{\mathrm{Kura}}$ fibers. This observation provides further evidence for the presence of long chain polyethylene waxes that was associated with the hydrophobic surface coating present on the $\mathrm{PVA}_{\text {Kura }}$ fibers by Scheffler et al., ${ }^{28}$ while the $\mathrm{PVA}_{\mathrm{Mini}}$ fibers were uncoated. The addition of this hydrophobic coating onto hydrophilic PVA fibers has reportedly increased the tensile strain capacity of PVA-reinforced Portland cements 10-fold by reducing the affinity of hydrophilic PVA fibers and enhancing frictional sliding. ${ }^{28-30,44}$ This approach of coating fibers to tailor the fiber-matrix interfacial properties has also been applied for CPCs. Specifically, dip-coating chitosan fibers in gelatin $^{45}$ or collagen ${ }^{46,47}$ has been successful at improving the mechanical performance of CPCs, where the compressive strength reportedly increased almost 2 -fold ${ }^{47}$ and the compressive modulus reached $\sim 1 \mathrm{GPa}$ which is comparable to that of wet canine bone. ${ }^{46}$

For both $\mathrm{CPC} / \mathrm{PVA}_{\mathrm{Mini}}$ and $\mathrm{CPC} / \mathrm{PVA}_{\mathrm{Kura}}$ cements, the flexural strength and toughness improve when the fiber length and concentration are increased. This trend has also been observed for different fiber types such as carbon, ${ }^{25,42}$ PLGA, ${ }^{48,49}$ aramid, and polyglactin, ${ }^{42}$ among others. ${ }^{2,17}$ Therefore, optimizing the stress transfer during frictional sliding is also dictated by the dimensions of the fibers. An effective transfer of loads from the matrix to fibers can only be achieved when fibers have a certain critical length which is determined by the aspect ratio of the fibers. ${ }^{26}$ If fibers are of insufficient length, then their lateral surface becomes too small and the transfer of loads is inhibited. ${ }^{2}$ This principle explains why the overall mechanical performance of CPCs reinforced with $3 \mathrm{~mm}$ fibers was inferior compared to that of $6 \mathrm{~mm}$ fibers, since reducing the fiber length from 6 to $3 \mathrm{~mm}$ causes a 2 -fold reduction in the aspect ratio (Table 1 ). However, this trend is only observed for CPC/PVA $\mathrm{Pura}_{\text {ra }}$ cements. Instead, CPC/ $\mathrm{PVA}_{\mathrm{Mini}}$ cements experience a practical upper limit where the strength and toughness start to plateau off upon incorporation of $5 \mathrm{wt} \%$ of $3 \mathrm{~mm}$ long fibers into the matrix. This practical upper limit can be attributed to several factors. First, poor transfer of loads due to nonoptimized fiber-matrix interfacial properties implicates that more fibers are needed to improve the mechanical properties of the cement. However, the use of such large quantities of fibers can cause fiber aggregation and inhomogeneous fiber dispersion. ${ }^{4,34,49}$ Poor fiber dispersion and the formation of fiber aggregates is especially evident for CPCs containing $5 \mathrm{wt} \% \mathrm{PVA}_{\text {Mini }}$ fibers $6 \mathrm{~mm}$ in length (Movie $\mathrm{S} 4 \mathrm{~A}, \mathrm{~B})$. We speculate that the introduction of shear forces during the mixing of the cement composite contributed to this heterogeneous fiber dispersion. Second, fibers with a low elastic modulus cannot carry higher loads ${ }^{2}$ since greater differences between the elastic modulus of the fiber and matrix cause additional strain at the interface, primarily in the less stiff PVA $_{\text {Mini }}$ fibers. ${ }^{17}$ Limitations on the mechanical performance of $\mathrm{CPC} / \mathrm{PVA}_{\mathrm{Mini}}$ cements can also be associated to the critical fiber embedment length $\left(l_{\mathrm{C}}\right)$, which is inversely proportional to the fiber-matrix interfacial shear strength and dependent on the fiber diameter. ${ }^{2}$ Since the diameter of $\mathrm{PVA}_{\mathrm{Mini}}$ fibers is nearly half that of PVA $_{\text {Kura }}$ fibers (Table 1), 6 mm PVA fibers might have surpassed their $l_{C}$, thereby limiting any improvement in the mechanical properties of CPCs. This is further supported by the fact that $6 \mathrm{~mm} \mathrm{PVA}_{\mathrm{Kura}}$ fibers have an aspect ratio that is nearly half that of $6 \mathrm{~mm}$ PVA $_{\mathrm{Mini}}$ fibers (Table 1), yet they are still capable of more than doubling the toughness of CPCs. This behavior can therefore be attributed to two factors: (i) the improved fiber-matrix interfacial properties of the hydrophobically coated PVA $_{\text {Kura }}$ fibers and (ii) the dimensions of the PVA $_{\text {Mini }}$ fibers. Therefore, tailoring and optimizing the fibers' aspect ratio, $l_{\mathrm{C}}$, and affinity to the matrix is of utmost importance when developing fiberreinforced CPCs for load-bearing applications. ${ }^{2,17,26}$

Finally, the addition of PVA fibers caused only minimal decreases in the flexural modulus of CPCs, irrespective of the type, length, or concentration. This is consistent with findings of Burguera et al. ${ }^{48}$ who reported only minimal reductions in the flexural modulus of CPC reinforced with $25 \% 8 \mathrm{~mm}$ PLGA yarns. They attribute this slight reduction in the flexural modulus to the fact that the PLGA fibers had a lower elastic modulus than the CPC matrix, which has also been observed elsewhere ${ }^{7,50}$ and is consistent with our findings. Moreover, the addition of PLGA microparticles not only contributed to a significant reduction in the flexural modulus but also the flexural strength and toughness, as shown in Figure 5. This reduction in flexural modulus is caused by the same phenomenon observed for the PVA fibers, where the PLGA microparticles have a lower modulus of elasticity than the CPC. However, the amount of PLGA microparticles introduced into the composite is significantly greater than that of the fibers (i.e., maximum of $40 \mathrm{wt} \%$ PLGA versus $5 \mathrm{wt}$ $\%$ of fibers). Therefore, this effect is more pronounced for PLGA-containing CPCs. Further, the spherical shape of PLGA microparticles corresponds to a low aspect ratio, which impedes any reinforcing effect. The reduction in flexural strength and toughness caused by the introduction of PLGA 
microparticles suggests that PLGA microparticles act as defect sites within the cement which facilitate crack formation and propagation.

In order to evaluate the biological performance of the newly developed PVA fiber-reinforced CPC, extensive in vitro and in vivo studies were performed. As the biocompatibility of the employed CPC matrix was confirmed numerous times in previous studies, ${ }^{51-54}$ an in vitro cytocompatibility assay was performed to understand the effect of PVA $_{\text {Kura }}$ or $\mathrm{PVA}_{\mathrm{Mini}}$ fibers themselves on osteoblast-like cells. ${ }^{55}$ The results showed that, for all selected concentrations, no significant differences between the control and the experimental groups were observed with up to $72 \mathrm{~h}$ of culture (Figure 4 ). These findings suggest that potential release of compounds from both types of PVA fibers did not affect the proliferation of the cultured cells up to $72 \mathrm{~h}$. However, the proliferation of cells exposed to $\mathrm{PVA}_{\text {Kura }}$ fibers was significantly reduced as compared to the fiber-free control after $168 \mathrm{~h}$ of culture, which could have been caused by the release of cytotoxic components from the hydrophobic surface coating and/or dimethyl sulfoxide from the core of the $\mathrm{PVA}_{\mathrm{Kura}}$ fibers, ${ }^{56}$ which is a cytotoxic organosulfur compound that is present in PVA $_{\text {Kura }}$ fibers up to $1 \%$. However, the surface morphology of both types of PVA fibers was not affected by extended cell culture (Figure S2). Due to the superior cytocompatibility of $\mathrm{PVA}_{\mathrm{Mini}}$ fibers, PVA $_{\text {Mini }}$ fibers were selected for further in vivo studies on the osteocompatibility of PVA fiber-reinforced CPCs.

Our histological data confirmed the osteocompatibility of all implanted groups (Figure 6), irrespective of the presence or absence of PVA $_{\text {Mini }}$ fibers. Apatitic CPCs are recognized as osteocompatible biomaterials $s^{5,58}$ due to the fact that they transform into nanocrystalline hydroxyapatite, the main inorganic component present in native bone. ${ }^{1-5,57}$ Generally, degradation of CPCs is facilitated by their intrinsic porosity. In a previous study conducted by our group, Lodoso-Torrecilla et al. ${ }^{59}$ measured the porosity of a CPC with a similar formulation using helium pycnometry and reported a total porosity of $48 \%$. We also calculated the theoretical porosity of our fiber-free CPC formulation to be $54 \%$, assuming the density of hydroxyapatite to be $3.156 \mathrm{~g} / \mathrm{mL}$. Therefore, we are confident that our CPCs have a porosity range between $45 \%$ and 55\%. However, apatite-forming CPC has a very low solubility at a physiological $\mathrm{pH}$ of 7.4 , which hampers the degradation of the implanted material and, consequently, the regeneration of the bone defect. A common strategy to accelerate the degradation of CPCs is to include acidproducing PLGA microparticles in the CPC matrix. ${ }^{51-54}$ Shortly after implantation, the PLGA microparticles degrade, creating a macroporous structure in the CPC matrix, while the acidic lactic and glycolic acid byproducts of PLGA accelerate the dissolution of the surrounding CPC matrix. In our study, the addition of PLGA improved the degradation of the CPC to $\sim 50 \%$ of the original amount after 6 weeks and $\sim 70 \%$ after 12 weeks. These values are in accordance with previously reported studies. ${ }^{51}$ In addition, the inclusion of PLGA allowed gradual ingrowth of new bone into the interior of the implanted cement resulting in $\sim 20 \%$ and $\sim 30 \%$ replacement after 6 and 12 weeks, respectively (Figure 7B). Regarding the degradation of PVA fibers, it should be emphasized that both types of PVA fibers were crystalline (Figure 1B) and heavily cross-linked during their fabrication. Consequently, these fibers are not biodegradable, which was confirmed by the absence of any morphological changes of the PVA fibers after extensive cell culture (Figure S2) and the histology images in which PVA fibers can be clearly distinguished after 12 weeks of implantation (Figure 6).

Interestingly, the percentage of new bone formation inside the ROI of CPC/PLGA and CPC/PLGA/PVA Mini $_{\text {specimens }}$ is almost similar, which suggests that the presence of $\mathrm{PVA}_{\mathrm{Mini}}$ fibers does not affect the ingrowth of new bone. This observation was confirmed by a detailed investigation of the interphase between PVA fibers and newly formed bone, since an intimate contact was revealed between bone and the surface of $\mathrm{PVA}_{\mathrm{Mini}}$ fibers (Figure $6 \mathrm{~g} 1, \mathrm{~h} 1$ ). Nevertheless, agglomerations of $\mathrm{PVA}_{\mathrm{Mini}}$ fibers induced a mild inflammatory response (Figure 6H1) which can be explained by the fact that PVA, unlike hardened apatitic $\mathrm{CPC}$, is not an osteoconductive material, ${ }^{58}$ thereby facilitating the formation of fibrotic tissue. These results stress that dispersion of the PVA fibers should be improved to avoid this fibrotic tissue response. Alternatively, PVA fibers could also be functionalized with an osteoconductive layer such as hydroxyapatite ${ }^{58}$ or bioactive glass ${ }^{60}$ to improve the capacity of PVA fibers to bind to bone, as reported previously.

Finally, our findings showed that the amount of cracking of nonimplanted cements versus cements implanted for 6 and 12 weeks (Figure 7D) was comparable, which suggests that preset cements cracked during their synthesis, most likely due to shrinkage caused by excessive drying prior to implantation, a phenomenon that was previously observed in other types of cements $^{61}$ as well as CPCs. ${ }^{62}$ As before, CPC/PVA ${ }_{\text {Mini }}$ samples cracked significantly less than their fiber-free counterparts (Figure 7D), which confirms again that the inclusion of PVA fibers successfully arrested crack propagation.

\section{CONCLUSIONS}

Mechanical reinforcement of CPCs was successfully achieved by incorporating PVA fibers into the cement matrix. PVA fiberreinforced CPCs exhibited higher flexural strength and toughness values than reported in the literature for fiberreinforced CPCs, with this effect increasing with increasing fiber length and concentration. Moreover, CPCs reinforced with both PLGA porogens and PVA fibers combined the reinforcing effect of PVA fibers with the resorbability induced by PLGA microparticles, thereby allowing for ingrowth of bone into tough PVA fiber-reinforced CPCs. Therefore, it was concluded that the inclusion of PVA fibers into nonresorbable CPCs as well as PLGA-containing resorbable CPCs strongly improved their strength and toughness without compromising their osteocompatibility. These findings render PVA fiberreinforced CPCs clinically relevant for applications in loadbearing skeletal sites.

\section{ASSOCIATED CONTENT}

\section{S Supporting Information}

The Supporting Information is available free of charge on the ACS Publications website at DOI: 10.1021/acsbiomaterials. 9 b00226.

Semiquantitative scoring system of the local biological response after implantation, mechanical properties of CPC composites obtained from three-point bending tests, scanning electron micrographs, and Fourier transform infrared spectra (PDF)

Movie S1 showing 3D reconstructed NanoCT video of a CPC/PLGA specimen (MP4) 
Movie S2 showing 3D reconstructed NanoCT video of a $\mathrm{CPC} / \mathrm{PLGA} / \mathrm{PVA}_{\mathrm{Mini}}$ specimen containing a fiber concentration of $2.5 \mathrm{wt} \%$ with a fiber length of $6 \mathrm{~mm}$ (MP4)

Movie S3A showing 3D reconstructed NanoCT video of a $\mathrm{CPC} / \mathrm{PVA}_{\mathrm{Mini}}$ specimen containing a fiber concentration of $2.5 \mathrm{wt} \%$ with a fiber length of $6 \mathrm{~mm}$, with the $\mathrm{CPC}$ matrix in blue and the fibers in green (MP4) Movie S3B showing the fibers alone in green (MP4) Movie S4A showing 3D reconstructed NanoCT video of a $\mathrm{CPC} / \mathrm{PVA}_{\mathrm{Mini}}$ specimen containing a fiber concentration of 5 wt \% with a fiber length of $6 \mathrm{~mm}$ with the $\mathrm{CPC}$ matrix in blue and the fibers in green (MP4) Movie S4A showing the fibers alone in green (MP4)

\section{AUTHOR INFORMATION}

\section{Corresponding Author}

*E-mail: sander.leeuwenburgh@radboudumc.nl. Phone: +31 (0)6 15409006.

\section{ORCID}

Jeroen J. J. P. van den Beuken: 0000-0003-2851-6896

Yi Zuo: 0000-0003-2301-9786

Sander C. G. Leeuwenburgh: 0000-0003-1471-6133

\section{Author Contributions}

${ }^{\|}$N.W.K. and S.d.L.S. contributed equally to this manuscript. The manuscript was written by through contributions from of all authors. All authors have given approval of to the final version of the manuscript.

\section{Funding}

This project was funded by The Netherlands Organization for Scientific Research (NWO; VIDI Grant 13455).

\section{Notes}

The authors declare no competing financial interest.

\section{ACKNOWLEDGMENTS}

The authors would like to thank our collaborators at KU Leuven for the nanoCT images which were produced at the Xray computed tomography facility of the Department of Development and Regeneration of the KU Leuven, financed by the Hercules foundation. The authors would like to acknowledge the Microscopic Imaging Centre (MIC) of the Radboud Institute for Molecular Life Sciences for providing access and instruction to obtain digital histological images used for this study. Lastly, the authors would also like to acknowledge their colleagues Vincent Cuijpers, Natasja van Dijk and Daniela Petre for their help in the acquisition of nanoCT images and movies, histological processing and acquisition of PVA fibers for this study, respectively.

\section{REFERENCES}

(1) Ginebra, M. P.; Traykova, T.; Planell, J. A. Calcium phosphate cements: Competitive drug carriers for the musculoskeletal system? Biomaterials 2006, 27 (10), 2171-2177.

(2) Krüger, R.; Groll, J. Fiber reinforced calcium phosphate cements - On the way to degradable load bearing bone substitutes? Biomaterials 2012, 33 (25), 5887-5900.

(3) Zhang, J.; Liu, W.; Schnitzler, V.; Tancret, F.; Bouler, J.-M. Calcium phosphate cements for bone substitution: Chemistry, handling and mechanical properties. Acta Biomater. 2014, 10 (3), $1035-1049$

(4) Zuo, Y.; Yang, F.; Wolke, J. G. C.; Li, Y.; Jansen, J. A. Incorporation of biodegradable electrospun fibers into calcium phosphate cement for bone regeneration. Acta Biomater. 2010, 6 (4), 1238-1247.

(5) Fu, Q.; Saiz, E.; Rahaman, M. N.; Tomsia, A. P. Toward strong and tough glass and ceramic scaffolds for bone repair. Adv. Funct. Mater. 2013, 23 (44), 5461-5476.

(6) Ginebra, M. P.; Canal, C.; Espanol, M.; Pastorino, D.; Montufar, E. B. Calcium phosphate cements as drug delivery materials. Adv. Drug Delivery Rev. 2012, 64 (12), 1090-1110.

(7) Gorst, N. J. S.; Perrie, Y.; Gbureck, U.; Hutton, A. L.; Hofmann, M. P.; Grover, L. M.; Barralet, J. E. Effects of fibre reinforcement on the mechanical properties of brushite cement. Acta Biomater. 2006, 2 (1), 95-102.

(8) Blattert, T. R.; Jestaedt, L.; Weckbach, A. Suitability of a calcium phosphate cement in osteoporotic vertebral body fracture augmentation: A controlled, randomized, clinical trial of balloon kyphoplasty comparing calcium phosphate versus polymethylmethacrylate. Spine 2009, 34 (2), 108-114.

(9) Lewis, G. Injectable bone cements for use in vertebroplasty and kyphoplasty: State-of-the-art review. J. Biomed. Mater. Res., Part B 2006, 76B (2), 456-468.

(10) Brown, W. E.; Chow, L. C. Dental resptorative cement pastes. United States Patent 4518430, 1985.

(11) Bohner, M.; Gbureck, U.; Barralet, J. E. Technological issues for the development of more efficient calcium phosphate bone cements: A critical assessment. Biomaterials 2005, 26 (33), 6423-6429.

(12) Canal, C.; Ginebra, M. P. Fibre-reinforced calcium phosphate cements: A review. J. Mech. Behav. Biomed. Mater. 2011, 4 (8), 16581671.

(13) Larsson, S.; Hannink, G. Injectable bone-graft substitutes: Current products, their characteristics and indications, and new developments. Injury 2011, 42, S30-S34.

(14) Tarsuslugil, S. M.; O’Hara, R. M.; Dunne, N. J.; Buchanan, F. J.; Orr, J. F.; Barton, D. C.; Wilcox, R. K. Development of calcium phosphate cement for the augmentation of traumatically fractured porcine specimens using vertebroplasty. J. Biomech. 2013, 46 (4), $711-715$.

(15) Sprio, S.; Guicciardi, S.; Dapporto, M.; Melandri, C.; Tampieri, A. Synthesis and mechanical behavior of $\beta$-tricalcium phosphate/ titania composites addressed to regeneration of long bone segments. J. Mech. Behav. Biomed. Mater. 2013, 17, 1-10.

(16) Dos Santos, L. A.; De Oliveira, L. C.; Da Silva Rigo, E. C.; Garcia Carrodeguas, R.; Ortega Boschi, A.; Celso Fonseca de Arruda, A. Fiber reinforced calcium phosphate cement. Artif. Organs 2000, 24 (3), 212-216.

(17) Geffers, M.; Groll, J.; Gbureck, U. Reinforcement strategies for load-bearing calcium phosphate biocements. Materials 2015, 8 (5), 2700-2717.

(18) O’Hara, R. M.; Orr, J. F.; Buchanan, F. J.; Wilcox, R. K.; Barton, D. C.; Dunne, N. J. Development of a bovine collagen-apatitic calcium phosphate cement for potential fracture treatment through vertebroplasty. Acta Biomater. 2012, 8 (11), 4043-4052.

(19) Dos Santos, L. A.; García Carrodeguas, R.; Ortega Boschi, A.; Celso Fonseca de Arruda, A. Dual-setting calcium phosphate cement modified with ammonium polyacrylate. Artif. Organs 2003, 27 (5), 412-418.

(20) Wang, J.; Liu, C.; Liu, Y.; Zhang, S. Double-network interpenetrating bone cement via in situ hybridization protocol. Adv. Funct. Mater. 2010, 20 (22), 3997-4011.

(21) Beaudoin, J. J. Handbook of Fiber-Reinforced Concrete. Principles, Properties, Developments and Applications; Noyes Publications: Saddle River, NJ, 1990.

(22) Xu, H. H. K.; Sun, L.; Weir, M. D.; Takagi, S.; Chow, L. C.; Hockey, B. Effects of incorporating nanosized calcium phosphate particles on properties of whisker-reinforced dental composites. $J$. Biomed. Mater. Res., Part B 2007, 81B (1), 116-125.

(23) Zorn, K.; Vorndran, E.; Gbureck, U.; Müller, F. A. Reinforcement of a magnesium-ammonium-phosphate cement with calcium phosphate whiskers. J. Am. Ceram. Soc. 2015, 98 (12), 40284035 . 
(24) Maenz, S.; Hennig, M.; Mühlstädt, M.; Kunisch, E.; Bungartz, M.; Brinkmann, O.; Bossert, J.; Kinne, R. W.; Jandt, K. D. Effects of oxygen plasma treatment on interfacial shear strength and post-peak residual strength of a PLGA fiber-reinforced brushite cement. J. Mech. Behav. Biomed. Mater. 2016, 57, 347-358.

(25) Xu, H. H. K.; Eichmiller, F. C.; Barndt, P. R. Effects of fiber length and volume fraction on the reinforcement of calcium phosphate cement. J. Mater. Sci.: Mater. Med. 2001, 12 (1), 57-65.

(26) Polini, A.; Petre, D. G.; Iafisco, M.; de Lacerda Schickert, S.; Tampieri, A.; Van den Beucken, J. J. J. P.; Leeuwenburgh, S. C. G. Polyester fibers can be rendered calcium phosphate-binding by surface functionalization with bisphosphonate groups. J. Biomed. Mater. Res., Part A 2017, 105 (8), 2335-2342.

(27) Ritchie, R. O. The conflicts between strength and toughness. Nat. Mater. 2011, 10, 817.

(28) Scheffler, C.; Zhandarov, S.; Jenschke, W.; Mäder, E. Poly (vinyl alcohol) fiber reinforced concrete: investigation of strain rate dependent interphase behavior with single fiber pullout test under quasi-static and high rate loading. J. Adhes. Sci. Technol. 2013, 27 (4), 385-402.

(29) Li, V. C.; Wang, S.; Wu, C. Tensile strain-hardening behavior of polyvinyl alcohol engineered cementitious composite (PVA-ECC). ACI Mater. J. 2001, 98 (6), 483-492.

(30) Li, V. C.; Wu, C.; Wang, S.; Ogawa, A.; Saito, T. Interface tailoring for strain-hardening polyvinyl alcohol-engineered cementitious composite (PVA-ECC). Mater. J. 2002, 99 (5), 463-472.

(31) Tummala, G. K.; Rojas, R.; Mihranyan, A. Poly (vinyl alcohol) hydrogels reinforced with nanocellulose for ophthalmic applications: general characteristics and optical properties. J. Phys. Chem. B 2016, 120 (51), 13094-13101.

(32) Baker, M. I.; Walsh, S. P.; Schwartz, Z.; Boyan, B. D. A review of polyvinyl alcohol and its uses in cartilage and orthopedic applications. J. Biomed. Mater. Res., Part B 2012, $100 B$ (5), 14511457.

(33) Ambrosio, L.; Guarino, V.; Sanginario, V.; Torricelli, P.; Fini, M.; Ginebra, M.; Planell, J.; Giardino, R. Injectable calciumphosphate-based composites for skeletal bone treatments. Biomed. Mater. 2012, 7 (2), 024113.

(34) Li, V. C.; Mishra, D. K.; Wu, H.-C. Matrix design for pseudostrain-hardening fibre reinforced cementitious composites. Mater. Constr. 1995, 28 (10), 586-595.

(35) Grosfeld, E.-C.; Hoekstra, J. W. M.; Herber, R.-P.; Ulrich, D. J. O.; Jansen, J. A.; Van den Beucken, J. J. J. P. Long-term biological performance of injectable and degradable calcium phosphate cement. Biomed. Mater. 2017, 12 (1), 015009.

(36) Liao, H.; Félix Lanao, R. P.; Van den Beucken, J. J. J. P.; Zhou, N.; Both, S. K.; Wolke, J. G. C.; Jansen, J. A. Size matters: effects of PLGA-microsphere size in injectable CPC/PLGA on bone formation. J. Tissue Eng. Regener. Med. 2016, 10 (8), 669-678.

(37) Félix Lanao, R. P.; Leeuwenburgh, S. C. G.; Wolke, J. G. C.; Jansen, J. A. Bone response to fast-degrading, injectable calcium phosphate cements containing PLGA microparticles. Biomaterials 2011, 32 (34), 8839-8847.

(38) Su, W.-Y.; Chen, Y.-C.; Lin, F.-H. A new type of biphasic calcium phosphate cement as a gentamicin carrier for osteomyelitis. Evidence-Based Complementary and Alternative Medicine 2013, 2013, 1.

(39) Ricciardi, R.; Auriemma, F.; De Rosa, C.; Lauprêtre, F. X-ray diffraction analysis of poly (vinyl alcohol) hydrogels, obtained by freezing and thawing techniques. Macromolecules 2004, 37 (5), 19211927.

(40) Fu, Q.; Saiz, E.; Rahaman, M. N.; Tomsia, A. P. Bioactive glass scaffolds for bone tissue engineering: state of the art and future perspectives. Mater. Sci. Eng., C 2011, 31 (7), 1245-1256.

(41) Karihaloo, B. L.; Wang, J. Micromechanical modelling of strain hardening and tension softening in cementitious composites. Comput. Mech. 1997, 19 (6), 453-462.

(42) Xu, H. H. K.; Eichmiller, F. C.; Giuseppetti, A. A. Reinforcement of a self-setting calcium phosphate cement with different fibers. J. Biomed. Mater. Res. 2000, 52 (1), 107-114.
(43) Boehm, A. V.; Meininger, S.; Tesch, A.; Gbureck, U.; Müller, F. A. The mechanical properties of biocompatible apatite bone cement reinforced with chemically activated carbon fibers. Materials 2018, 11 (2), 192.

(44) Li, V. C. On engineered cementitious composites (ECC). J. Adv. Concr. Technol. 2003, 1 (3), 215-230.

(45) Pan, Z.; Jiang, P.; Fan, Q.; Ma, B.; Cai, H. Mechanical and biocompatible influences of chitosan fiber and gelatin on calcium phosphate cement. J. Biomed. Mater. Res., Part B 2007, 82B (1), 246252.

(46) Lian, Q.; Li, D.; Jin, Z.; Wang, J.; Li, A.; Wang, Z.; Jin, Z. Fabrication and in vitro evaluation of calcium phosphate combined with chitosan fibers for scaffold structures. J. Bioact. Compat. Polym. 2009, 24 (1_suppl), 113-124.

(47) Lian, Q.; Li, D. C.; He, J. K.; Wang, Z. Mechanical properties and in-vivo performance of calcium phosphate cement-chitosan fibre composite. Proc. Inst. Mech. Eng., Part H 2008, 222 (3), 347353.

(48) Burguera, E. F.; Xu, H. H. K.; Takagi, S.; Chow, L. C. High early strength calcium phosphate bone cement: effects of dicalcium phosphate dihydrate and absorbable fibers. J. Biomed. Mater. Res., Part A 2005, 75A (4), 966-975.

(49) Zhang, Y.; Xu, H. H. K. Effects of synergistic reinforcement and absorbable fiber strength on hydroxyapatite bone cement. J. Biomed. Mater. Res., Part A 2005, 75A (4), 832-840.

(50) Xu, H. H. K.; Simon Jr, C. G. Self-hardening calcium phosphate composite scaffold for bone tissue engineering. J. Orthop. Res. 2004, 22 (3), 535-543.

(51) Bao, C.; Chen, W.; Weir, M. D.; Thein-Han, W.; Xu, H. H. K. Effects of electrospun submicron fibers in calcium phosphate cement scaffold on mechanical properties and osteogenic differentiation of umbilical cord stem cells. Acta Biomater. 2011, 7 (11), 4037-4044.

(52) Wu, T. Y.; Zhou, Z. B.; He, Z. W.; Ren, W. P.; Yu, X. W.; Huang, Y. Reinforcement of a new calcium phosphate cement with RGD-chitosan-fiber. J. Biomed. Mater. Res., Part A 2014, 102 (1), 6875.

(53) Xu, H. H. K.; Simon, C. G. Self-hardening calcium phosphate cement-mesh composite: Reinforcement, macropores, and cell response. J. Biomed. Mater. Res. 2004, 69A (2), 267-278.

(54) Zhao, L.; Burguera, E. F.; Xu, H. H. K.; Amin, N.; Ryou, H.; Arola, D. D. Fatigue and human umbilical cord stem cell seeding characteristics of calcium phosphate-chitosan-biodegradable fiber scaffolds. Biomaterials 2010, 31 (5), 840-847.

(55) Wang, M. O.; Etheridge, J. M.; Thompson, J. A.; Vorwald, C. E.; Dean, D.; Fisher, J. P. Evaluation of the in vitro cytotoxicity of cross-linked biomaterials. Biomacromolecules 2013, 14 (5), 13211329.

(56) Material Safety Data Sheet: Polyvinylalcohol; Kuraray Co., Ltd.: Osaka, Japan, 2015; p 3.

(57) Sariibrahimoglu, K.; An, J.; Van Oirschot, B. A. J. A.; Nijhuis, A. W. G.; Eman, R. M.; Alblas, J.; Wolke, J. G. C.; Van den Beucken, J. J. J. P.; Leeuwenburgh, S. C. G.; Jansen, J. A. Tuning the degradation rate of calcium phosphate cements by incorporating mixtures of polylactic-co-glycolic acid microspheres and glucono-delta-lactone microparticles. Tissue Eng., Part A 2014, 20 (21-22), 2870-2882.

(58) Sheikh, F. A.; Barakat, N. A. M.; Kanjwal, M. A.; Park, S. J.; Park, D. K.; Kim, H. Y. Synthesis of poly (vinyl alcohol)(PVA) nanofibers incorporating hydroxyapatite nanoparticles as future implant materials. Macromol. Res. 2010, 18 (1), 59-66.

(59) Lodoso-Torrecilla, I.; Van Gestel, N. A. P.; Diaz-Gomez, L.; Grosfeld, E. C.; Laperre, K.; Wolke, J. G. C.; Smith, B. T.; Arts, J. J.; Mikos, A. G.; Jansen, J. A.; et al. Multimodal pore formation in calcium phosphate cements. J. Biomed. Mater. Res., Part A 2018, 106 (2), 500-509.

(60) Gao, C.; Gao, Q.; Li, Y.; Rahaman, M. N.; Teramoto, A.; Abe, $\mathrm{K}$. Preparation and in vitro characterization of electrospun PVA scaffolds coated with bioactive glass for bone regeneration. J. Biomed. Mater. Res., Part A 2012, 100A (5), 1324-1334. 
(61) Yang, B.; Zuo, Y.; Zou, Q.; Li, L.; Li, J.; Man, Y.; Li, Y. Effect of ultrafine poly ( $\varepsilon$-caprolactone) fibers on calcium phosphate cement: in vitro degradation and in vivo regeneration. Int. J. Nanomed. 2016, $11,163$.

(62) Baănt, Z. P.; Raftshol, W. J. Effect of cracking in drying and shrinkage specimens. Cem. Concr. Res. 1982, 12 (2), 209-226. 